\title{
16. OLIGOCENE TO MIOCENE STABLE ISOTOPE STRATIGRAPHY AND PLANKTONIC FORAMINIFER BIOSTRATIGRAPHY OF THE SIERRA LEONE RISE (DSDP SITE 366 AND ODP SITE 667) ${ }^{1}$
}

\author{
Kenneth G. Miller, ${ }^{2}$ James D. Wright, ${ }^{3}$ and Andrew N. Brower ${ }^{3}$
}

\begin{abstract}
We have integrated Oligocene to lower upper Miocene planktonic foraminifer biostratigraphy with benthic foraminifer (Cibicidoides spp.) stable isotope records for two sites drilled on opposite sides of the Sierra Leone Rise in the eastern equatorial Atlantic Ocean. Deep Sea Drilling Project Site 366 ( $2853 \mathrm{~m}$ present water depth; 2200-2800 m paleodepth) recovered an Oligocene to upper Miocene record with a minor unconformity in the "middle" Oligocene and a condensed middle Miocene section. Ocean Drilling Program Site 667 (3529 m present depth; 3000-3500 m paleodepth) recovered an apparently continuous "middle" Oligocene to lower middle Miocene record and a similar condensed middle Miocene section. The Oligocene to lower Miocene sections were deposited at similar sedimentation rates $(\sim 11-16 \mathrm{~m} / \mathrm{m} . \mathrm{y}$.$) .$

Stable isotope stratigraphy proved to be useful in establishing intra- and interbasinal correlations. In addition to the well-known earliest Oligocene and middle Miocene $\delta^{18} \mathrm{O}$ increases, a distinct $\delta^{18} \mathrm{O}$ increase occurred near the Oligocene/Miocene boundary. Carbon isotope variations provide similar potential for improving correlations; for example, a $\delta^{13} \mathrm{C}$ increase occurred near the Oligocene/Miocene boundary in concert with increased $\delta^{18} \mathrm{O}$ values. There was little $\delta^{13} \mathrm{C}$ difference between the western Atlantic and eastern Atlantic basins during the late Oligocene and most of the middle Miocene; in contrast, eastern basin $\delta^{13} \mathrm{C}$ values were slightly lower than those in the western basins during the earliest Oligocene (about 35-33 Ma) and early Miocene (about 22-18 Ma).
\end{abstract}

\section{BACKGROUND}

\section{Stable Isotope Stratigraphy}

With the recovery of relatively complete pelagic sections by the Deep Sea Drilling Project (DSDP) and the Ocean Drilling Program (ODP), stable isotope studies have become a standard means of evaluating Mesozoic-Cenozoic oceanographic and sea-level changes (e.g., Savin et al., 1975; Shackleton and Kennett, 1975; Miller et al., 1987b). Stable isotope studies also can be used to improve correlations because the fluctuating patterns can be matched on both local and global levels. This potential has been fully realized in Quaternary stratigraphy, yielding resolution as fine as 5 k.y. (e.g., Imbrie et al., 1984). Because of inherent uncertainties in establishing the age and synchrony of Cenozoic isotope events, the development of stable isotope records as a means of pre-Pleistocene correlation has lagged Quaternary studies.

Distinct oxygen and carbon isotope fluctuations occurred during the Cenozoic, providing a means of establishing equivalency among records. Initial uncertainties about the timing and distribution of these events limited their stratigraphic use. For example, Savin et al. (1975) and Shackleton and Kennett (1975) noted that a distinct increase in $\delta^{18} \mathrm{O}$ occurred near the end of the Eocene. A decade of detailed stratigraphic studies have shown that this increase occurred in the earliest Oligocene (about 35.9-35.7 Ma according to the time scale of Berggren et al., 1985a) in all oceans (Kennett and Shackleton, 1976; Keigwin, 1980; Miller et al., 1985c; see also the review by Vergnaud-Grazzini and Oberhänsli, 1986). The synchrony of this increase among oceans is no longer in doubt, and its age

\footnotetext{
${ }^{1}$ Ruddiman, W., Sarnthein, M., et al., 1989. Proc. ODP, Sci. Results, 108: College Station, TX (Ocean Drilling Program).

2 Department of Geological Sciences, Rutgers University, New Brunswick, NJ 08903, and Lamont-Doherty Geological Observatory, Palisades, NY 10964.

${ }^{3}$ Lamont-Doherty Geological Observatory and Department of Geological Sciences, Columbia University, Palisades, NY 10964.
}

has been firmly established by first-order calibration to the Geomagnetic Polarity Time Scale (GPTS) (Oberhänsli and Toumarkine, 1985; Miller et al., 1988). Other pre-Quaternary isotopic events (e.g., the middle Miocene $\delta^{18} \mathrm{O}$ increases, the late early to early middle Miocene $\delta^{13} \mathrm{C}$ increase, the late Miocene "carbon shift," and the latest Miocene $\delta^{18} \mathrm{O}$ increases) have been shown to be synchronous within the uncertainties of biostratigraphy and therefore are useful in chronostratigraphy (Haq et al., 1980; Vincent and Berger, 1985; Hodell and Kennett, 1986; Keigwin, 1987; among others).

Cenozoic isotope correlations are useful in two frequency bands. First, high-resolution $\left(10^{4}-10^{5} \mathrm{yr}\right)$ Pliocene $\delta^{18} \mathrm{O}$ studies have delineated isotope stages similar to those in the Quaternary (Ruddiman et al., 1986). High-resolution Miocene isotope studies also show promise (Pisias et al., 1985; Keigwin, 1987; Keigwin et al., 1987); future detailed studies may lead to high-resolution isotope correlations in Miocene and older sections. Second, $\delta^{18} \mathrm{O}$ variations on the $0.5-1.0 \mathrm{~m} . \mathrm{y}$. scale can be used to improve correlations (e.g., Miller et al., 1987a, $1987 \mathrm{c})$. The carbon isotope record also provides potential correlations (e.g., Vincent and Berger, 1985; Shackleton, 1987). Since our primary goals involve carbon isotope comparisons, we have generally supplemented our biostratigraphy with $\delta^{18} \mathrm{O}$ stratigraphy alone to avoid possible circular reasoning.

Firm chronostratigraphic control is required to evaluate the use of stable isotopes in stratigraphy. Ideally, stable isotope records should be directly calibrated via magnetostratigraphy to the GPTS (e.g., Site 522, Miller et al., 1988); records from various oceans and hydrographic settings should be examined. This is not practical, for there are relatively few good magnetostratigraphic sections available. For example, there are only two reliable lower to middle Miocene magnetostratigraphic records (Fig. 1; Site 563, Miller et al., 1985b; Site 608, Clement and Robinson, 1987), and one (Site 563) contains unconformities that result in ambiguous magnetostratigraphy in certain intervals. Biostratigraphy can be used cautiously to evaluate the synchrony or diachrony of isotope fluctuations. Such biostratigraphic evaluations are useful at tropical and 


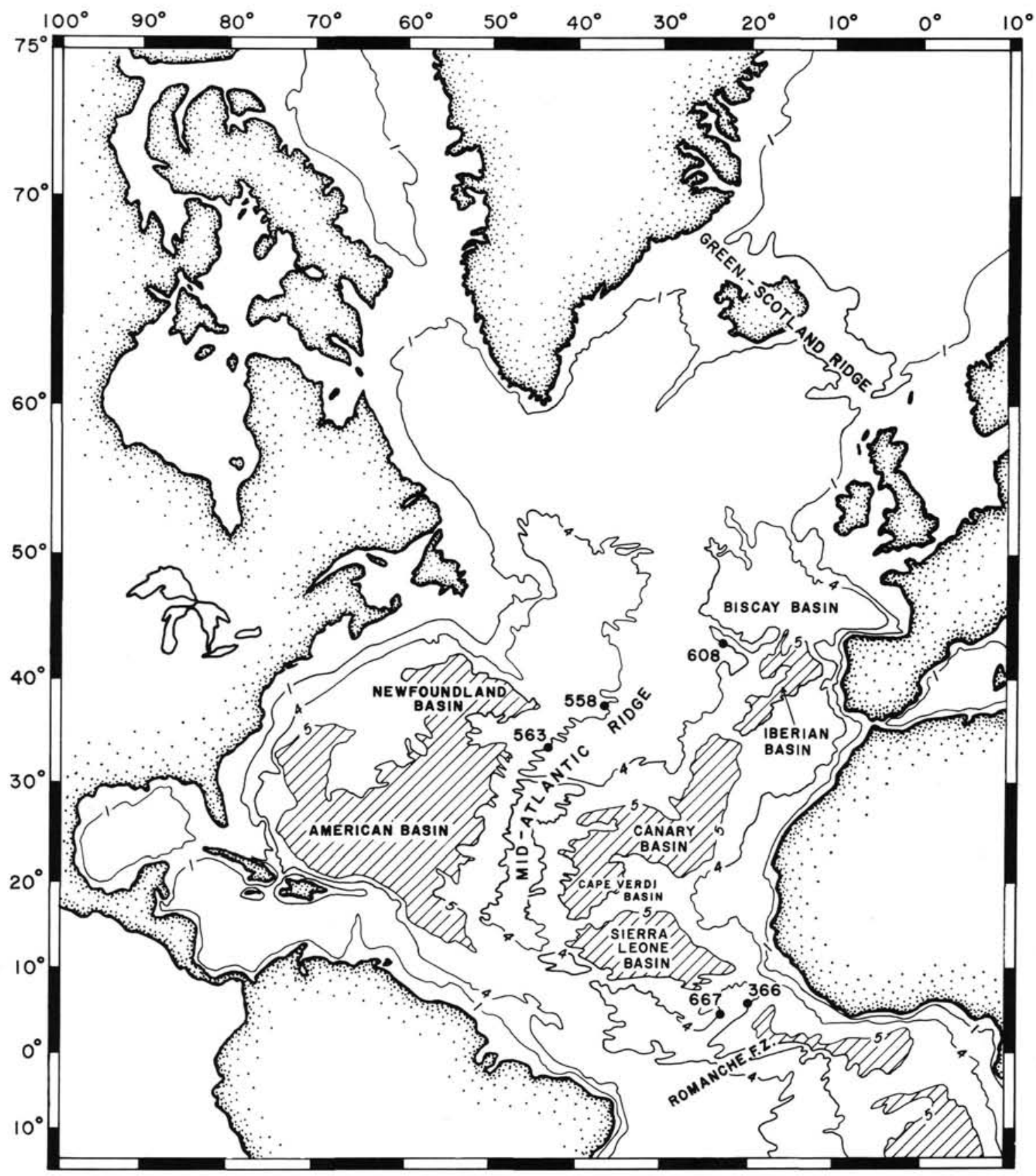

Figure 1. Location map showing Sites $366,558,563,608$, and 667 mentioned in the text; the 1-, 4-, and 5-km contour intervals are indicated and depths greater than $5 \mathrm{~km}$ are cross hatched. F.Z. = Fracture Zone. Modified after Deep Sea Drilling Project (unpubl.).

subtropical locations where standard biozonations are generally applicable, but $\delta^{18} \mathrm{O}$ correlations may be more reliable in higher latitudes because biostratigraphic first and last occurrences are often latitudinally diachronous (e.g., Johnson and Nigrini, 1985).

\section{Setting}

The eastern basins of the Atlantic are bounded to the south by the Walvis Ridge, to the north by the Iceland-Shetland Ridge, and to the west by the Mid-Atlantic Ridge (Fig. 1). Most of the modern deep water entering across the Iceland-
Shetland portion of the Greenland-Scotland Ridge is advected into the western basins and does not contribute directly to the ventilation of the deep eastern basins (Broecker and Peng, 1982). The eastern basins below approximately $3700 \mathrm{~m}$ are ventilated by advection of a mixture of $\sim 80 \%$ Northern Component/North Atlantic Deep Water (NC/NADW) and $\sim 20 \%$ Antarctic Bottom Water (AABW) through low-latitude fracture zones including the Romanche Fracture Zone (Metcalf et al., 1964; Broecker and Peng, 1982; Curry and Lohmann, 1983, 1985). Thus, NC/NADW today fills the eastern basins, constituting the primary deep-water mass above and below the sill. 
The Sierra Leone Rise provides a monitor of vertical deep-water variability in the eastern Atlantic above and below the present sill depth of the Romanche Fracture Zone $(\sim 3700$ m; Curry and Lohmann, 1983, 1985). From studies of piston cores obtained along the flanks of the Sierra Leone Rise, Curry and Lohmann $(1983,1985)$ noted that the eastern basins of the North Atlantic have alternated between two oceanographic modes during late Pleistocene glacial-interglacial cycles. During interglacials, a copious supply of NC/NADW to the eastern basins via the Romanche Fracture Zone corresponded with increased carbonate and decreased organic carbon accumulation. During glacials, a reduced supply of NC/NADW resulted in decreased oxygenation in the deep eastern basins, enhanced preservation of organic carbon, decreased carbonate accumulation, and increased opal accumulation (Curry and Lohmann, 1985). These studies are among those that have documented $\delta^{13} \mathrm{C}$ as a powerful tracer of Quaternary deep-water circulation (see also Shackleton et al., 1983; Mix and Fairbanks, 1985; Oppo and Fairbanks, 1987; among others).

The development of pre-Pleistocene North Atlantic deepwater circulation similarly can be evaluated using benthic foraminifer carbon isotope comparisons. A "glacial-type" circulation pattern during the Pliocene reduced the influence of NC/NADW on the eastern basins below the $3700-\mathrm{m}$ sill depth, although NC/NADW was produced during this interval (Curry and Miller, this vol.). Thus, this climatic control of the oxygenation of the deep eastern basins persisted for over 3 m.y. On the time scales considered here, however, tectonic changes in the configuration of the sills controlling flow into the eastern basins also must be considered (e.g., Bonatti and Chermak, 1981).

We have demonstrated that deep water formed in the North Atlantic and/or its marginal seas during the pre-
Pliocene. The western North Atlantic (Site 563; paleodepth $>2 \mathrm{~km}$; Fig. 1) was enriched in ${ }^{13} \mathrm{C}$ relative to the Pacific during the early Oligocene and much of the Miocene (Miller and Fairbanks, 1985). This indicates that the North Atlantic was proximal to a "young" deep-water source at these times similar to present-day NC/NADW (Miller and Fairbanks, 1985). We have documented that $\delta^{13} \mathrm{C}$ values at Iberian Basin Site 608 (Kings Trough; see Fig. 1) were slightly lower than at western basin Site 563 from $\sim 24$ to 16 Ma (early to early middle Miocene), reflecting less ventilation of the Kings Trough region (Miller et al., 1987a). The low ventilation noted at Site 608 may be attributed to either (1) local tectonic isolation, since Kings Trough is tectonically active (Kidd and Ramsay, 1987), or (2) changes in the ventilation of the eastern basins of the Atlantic (e.g., Sierra Leone, Cape Verde, Canary, Iberian, and Biscay; see Fig. 1) analogous to those of the Quaternary (Miller et al., 1987a).

One of the goals of Leg 108 was to obtain OligoceneHolocene records both above and below the present-day sill depth of the Romanche Fracture Zone $(\sim 3700 \mathrm{~m})$ to test for changes in hydrography due to climatic and/or tectonic controls. This region is also critical for understanding surfacewater changes (Curry and Crowley, 1987). Deep burial and diagenesis preclude meaningful stable isotope studies of Eocene sediments at the Sierra Leone Rise. Hence, the original goal of Leg 108 was to obtain Oligocene-Holocene sections from a wide bathymetric range on the rise, as was done for the Quaternary section with piston cores (Curry and Lohmann, 1983).

Because of time limitations, however, a continuously cored Oligocene-Miocene section was obtained only at Site 667, drilled in $3529 \mathrm{~m}$ present water depth on the southwest flank of the rise $\left(4^{\circ} 34.15^{\prime} \mathrm{N}, 21^{\circ} 54.68^{\prime} \mathrm{W}\right.$; see Figs. 1 and 2$)$. Leg 41 drilled Site 366 in $2853 \mathrm{~m}$ water depth on the northeast flank of

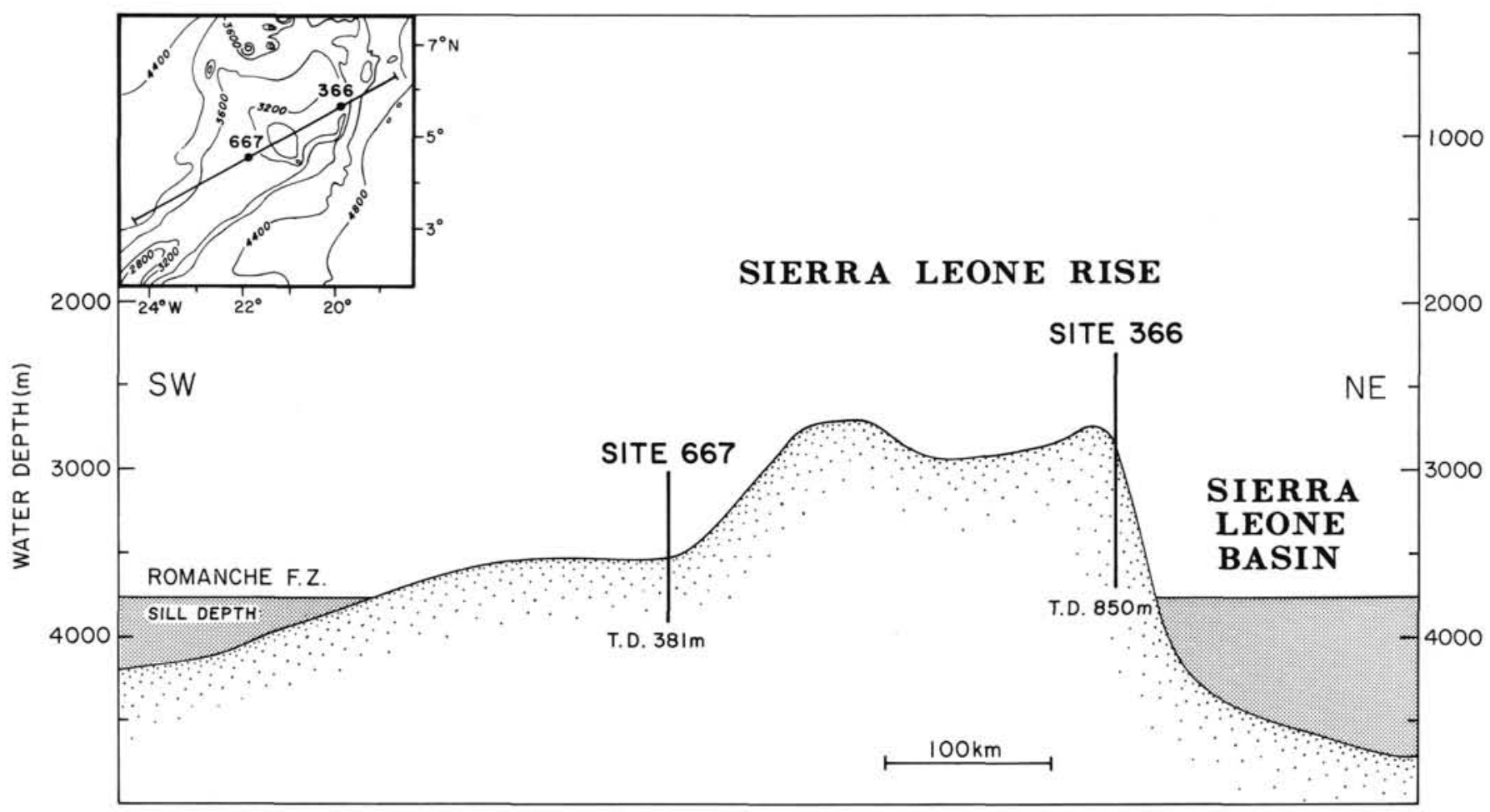

Figure 2. Generalized transect across the Sierra Leone Rise. Inset map shows location (modified after Fig. 1, p. 22, in Lancelot, Seibold, et al., 1978). Vertical exaggeration is 1:1000. The sill depth of the Romanche Fracture Zone is indicated, and depths below are stippled. T.D. $=$ total depth penetrated by borehole. 
Sierra Leone Rise

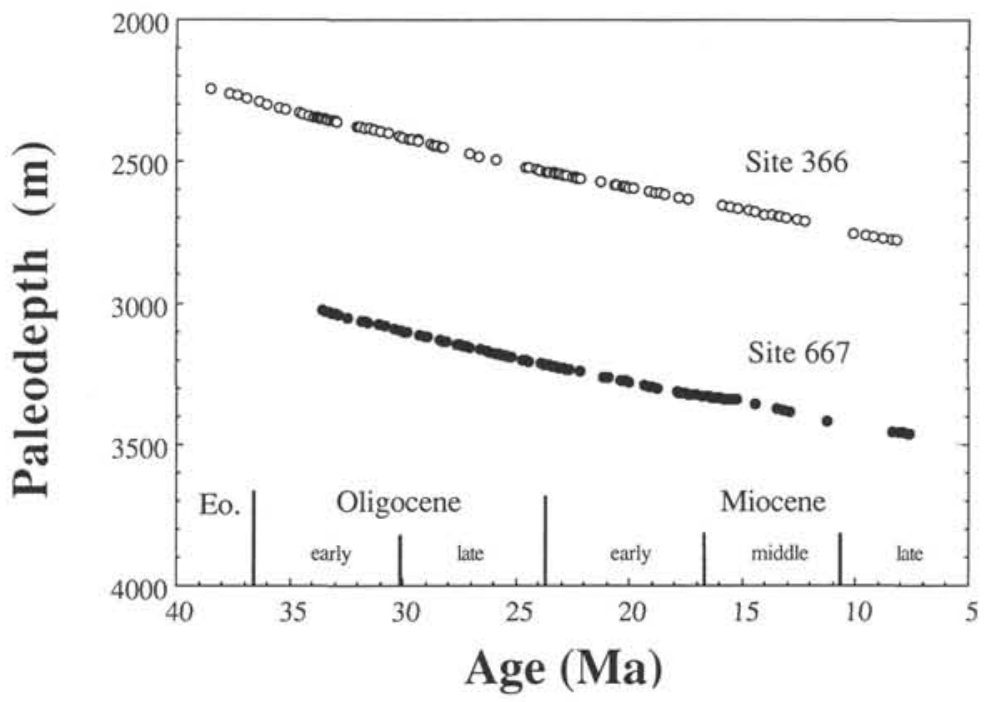

Figure 3. Age-paleodepth distribution for samples considered here (see "Methods" section, this chapter).

the rise $\left(5^{\circ} 40.7^{\prime} \mathrm{N}, 19^{\circ} 51.1^{\prime} \mathrm{W}\right.$; Lancelot, Seibold, et al., 1978) and obtained a relatively complete Oligocene-Miocene section (Figs. 1 and 2). These two Oligocene-Miocene records allow (1) the evaluation of vertical differences in bottom-water characteristics spanning approximately $700 \mathrm{~m}$ present depth and $800 \mathrm{~m}$ paleodepth (Figs. 2 and 3 ) and (2) the assessment of possible tectonic shallowing of the sill depth.

The sections obtained on the Sierra Leone Rise allow us not only to document eastern basin $\delta^{13} \mathrm{C}$ distribution, but also to apply Oligocene-Miocene isotope and biostratigraphy to the subequatorial locations. Magnetostratigraphic studies of Sites 366 and 667 were not successful because of low remanent magnetism, low paleoinclinations, and unoriented cores at Site 366 (Tauxe, this vol.). As a result, chronostratigraphic control must be obtained using biostratigraphy or other correlation techniques. We examined the Oligocene-Miocene sections at Sites 366 and 667 for planktonic foraminifer biostratigraphy to supplement the existing planktonic biostratigraphy (for Site 366, see Krasheninnikov and Pflaumann, 1978a, 1978b, and Keller, 1986; for Site 667, see Ruddiman, Sarnthein, et al., 1988, pp. 833-930). We used the foraminifer biostratigraphy and the available nannofossil biostratigraphy to correlate to the time scale of Berggren et al. (1985a, 1985b). These sections provide a basis for evaluating Oligocene-Miocene oceanographic conditions in this region (Figs. 2 and 3).

\section{METHODS}

\section{Biostratigraphic Methods}

We qualitatively examined 1-6 samples per core $(9.5 \mathrm{~m})$ to interpret planktonic foraminifer biostratigraphy (samples indicated on Figs. 4 and 5). Because of time limitations, we did not complete our biostratigraphic studies of the Miocene of Site 366 and relied upon the published biostratigraphy (Krasheninnikov and Pflaumann, 1978b). We determined first (FOs) and last (LOs) occurrences of diagnostic marker taxa (Figs. 4 and 5), but we did not evaluate the abundances of these taxa or attempt to describe the entire fauna. Taxonomic concepts follow Stainforth et al. (1975), Blow (1979), and Bolli and Saunders (1985), except as modified in the "Taxonomic and Stratigraphic Notes" (this chapter). The Miocene zonation is essentially that of Blow (1969). The Oligocene zonation is a hybrid of the tropical zonal schemes of Bolli (1957) and Blow (1979). Inasmuch as neither zonation has proven appropriate for the Paleogene, a formal recodi- fication has been proposed by Berggren and Miller 1988. The Oligocene portion of this revised zonation is:

Zone P18 is the interval from the LO of Turborotalia cerroazulensis s.l. to the LO of Pseudohastigerina spp.;

Zone P19 is the interval from the LO of Pseudohastigerina spp. to the LO of "Turborotalia" ampliapertura;

Zone P20 is the interval from the LO of " $T$." ampliapertura to the FO of Globigerina angulisuturalis;

Zone P21a is the interval from the FO of $G$. angulisuturalis to the LO of Chiloguembelina spp.;

Zone P21b is the interval from the LO of Chiloguembelina spp. to the LO of Paragloborotalia opima opima;

Zone P22 is the interval from the LO of $P$. opima opima to the FO of Globorotalia kugleri; and

the FO of Globorotalia kugleri is used to recognize the Oligocene/ Miocene boundary and the base of Zone N4.

Ages in millions of years (Ma) are assigned by linearly interpolating between biostratigraphic levels (Tables 1 through 5). Ages for the biostratigraphic datum levels are from Berggren et al. (1985a, 1985b).

\section{Stable Isotopes}

Samples for isotope and faunal analyses were soaked in hydrogen peroxide, washed with sodium hexametaphosphate in tap water through a $63-\mu \mathrm{m}$ sieve, and air dried. Foraminifers were ultrasonically cleaned in deionized and distilled water (10-20 s). Oxygen and carbon isotope studies were performed on the benthic foraminifer Cibicidoides spp. (Figs, 4 and 5; Tables 1 and 2), which were picked from the $>150-\mu \mathrm{m}$-size fraction and roasted at $370^{\circ} \mathrm{C}$ in a vacuum. Studies have shown that this taxon accurately records $\delta^{13} \mathrm{C}$ variations in seawater and is lower than $\delta^{18} \mathrm{O}$ equilibrium by about $0.64 \%$ (e.g., Shackleton and Opdyke, 1973; Graham et al., 1981). The $\mathrm{CaCO}_{3}$ was analyzed at Lamont-Doherty Geological Observatory by either a Carousel-48 automatic carbonate preparation device (R. Fairbanks, unpubl. data, 1988) attached to a Finnigan MAT 251 or by standard procedures (e.g., Fairbanks and Matthews, 1978) on a VG Micromass 903E.

\section{Paleobathymetry}

Paleodepth estimates were calculated assuming simple thermal subsidence and empirical age-subsidence curves (Sclater et al., 1971; Berger and Winterer, 1974; among others) of the form

$$
P d=I d+k t^{1 / 2}-S ; I d=P r-k(\text { age basement })^{1 / 2}+S,
$$




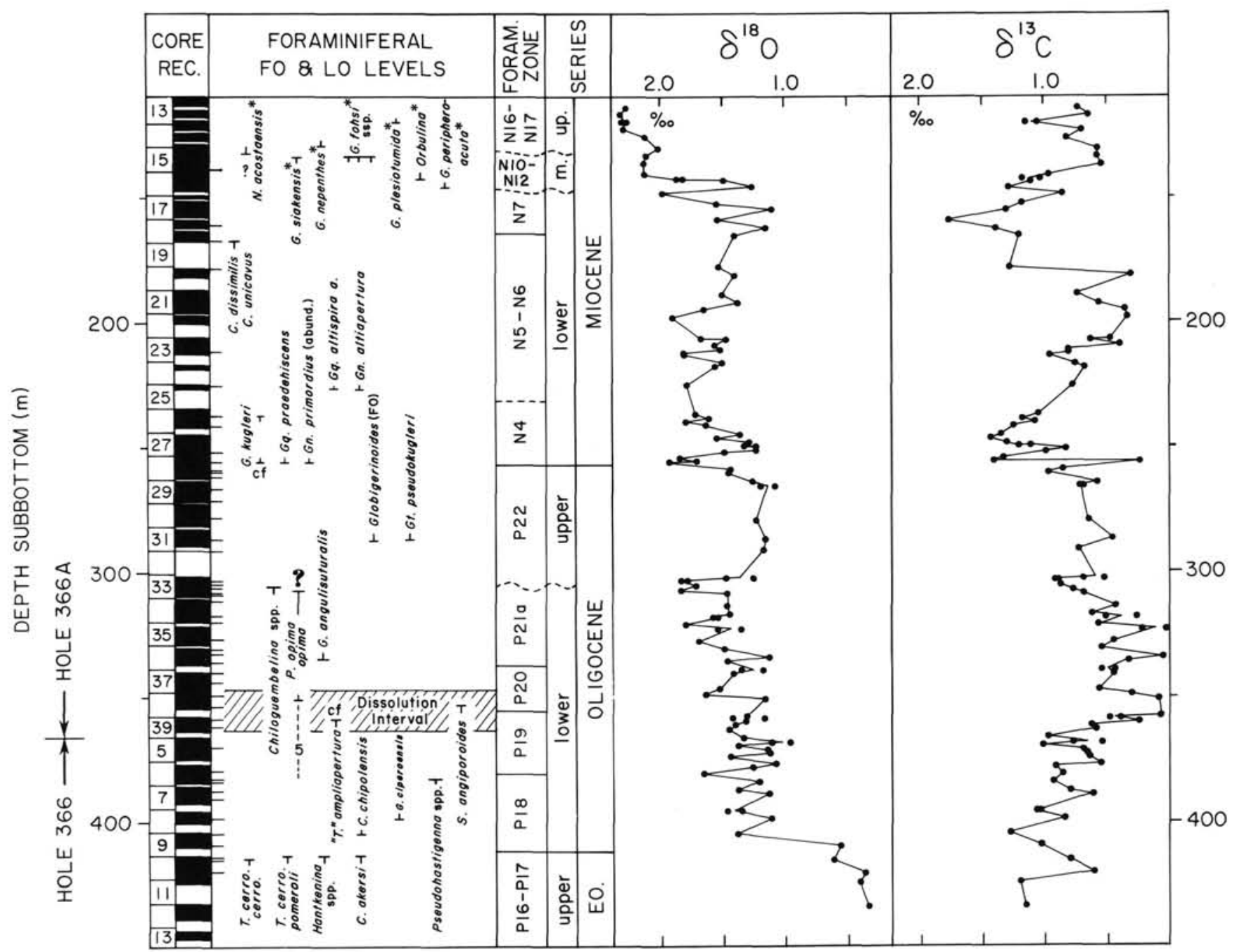

Figure 4. Biostratigraphy and stable isotope stratigraphy, Site 366. REC $=$ recovery. Shading indicates recovered intervals. FO $=$ first occurrences indicated with inverted T's; LO = last occurrences indicated with T's. Tick marks next to REC indicate samples examined for biostratigraphy. Duplicate isotope analyses are plotted, and a line is drawn through the mean value of the duplicates. $5=$ five-chambered forms only of $P$. opima opima; ${ }^{*}=$ datum levels after Krasheninnikov and Pflaumann (1978a). Zonation after this study.

for crust younger than $80 \mathrm{Ma}$, and

$P d=I d-A+A \times e^{(-t / \tau)}-S ; I d=P r+A-A \times e^{(\text {age basement } / \tau)}+S$,

for crust older than $80 \mathrm{Ma}$, where $P r=$ present depth; $P d=$ paleodepth; $I d=$ initial depth; $t=$ age basement minus the age of level considered; $S=$ sediment correction of $0.66 \times$ (basement depth sub-bottom minus depth sub-bottom of level considered); and $\tau=$ decay constant of 62.5 . The constants $A$ and $k$ have been empirically determined for the Atlantic as -3650 and 300 , respectively (Miller et al., 1987a).

We used the second two equations, assuming a crustal age of 80 Ma and $A=-3650$, to compute paleodepths for Sites 366 and 667 . Depth to basement at Site 366 was estimated to be approximately 914 $\mathrm{m}$, based upon a two-way traveltime of $0.93 \mathrm{~s}$ on the Challenger 48 profile (Lancelot, Siebold, et al., 1978, p. 42, fig. 13) and a velocity profile of approximately $1.75 \mathrm{~km} / \mathrm{s}$ for $0-500 \mathrm{~m}$ and $2.30 \mathrm{~km} / \mathrm{s}$ for depths $>500 \mathrm{~m}$ (Lancelot, Seibold, et al., 1978). Depth to basement was not determinable on the basis of the JOIDES Resolution Leg 108 approach line (Ruddiman, Sarnthein, et al., 1988); we used $914 \mathrm{~m}$, the depth to basement at Site 366, as a rough approximation. Oligocene water depths for Sites 366 and 667 were approximately $2300 \mathrm{~m}$ and $3100 \mathrm{~m}$, respectively; Miocene depths were approximately $2600 \mathrm{~m}$ and $3400 \mathrm{~m}$, respectively (Fig. 3).

\section{RESULTS}

\section{Biostratigraphy}

\section{Site 366}

Hole 366 penetrated Eocene and older sediments (Lancelot, Seibold, et al., 1978). We record the LO of planktonic foraminifer taxa used to recognize the Eocene/Oligocene boundary (Turborotalia cerroazulensis cerroazulensis and Hantkenina spp.; Berggren et al., 1985a) between Cores 41-366-9 and -10, in agreement with Keller (1986). We found no offset between the LO of these two taxa, agreeing with biostratigraphic relationships at eastern North Atlantic Site 549 (Snyder et al., 1985). In some sections (see summary in Pomerol and Premoli Silva, 1986), an offset of about 0.15 m.y. has been observed between the LO of $T$. cerroazulensis cerroazulensis and Hantkenina spp. The coincidence of these $\mathrm{LO}$ at Hole 366 may be due to a $\sim 2-\mathrm{m}$ coring gap between Cores 41-366-9 and -10 (Fig. 4).

The lower Oligocene, recognized by the interval between the LO of Hantkenina spp. and the LO of Chiloguembelina spp. (Berggren et al., 1985a), is approximately $106 \mathrm{~m}$ thick at 


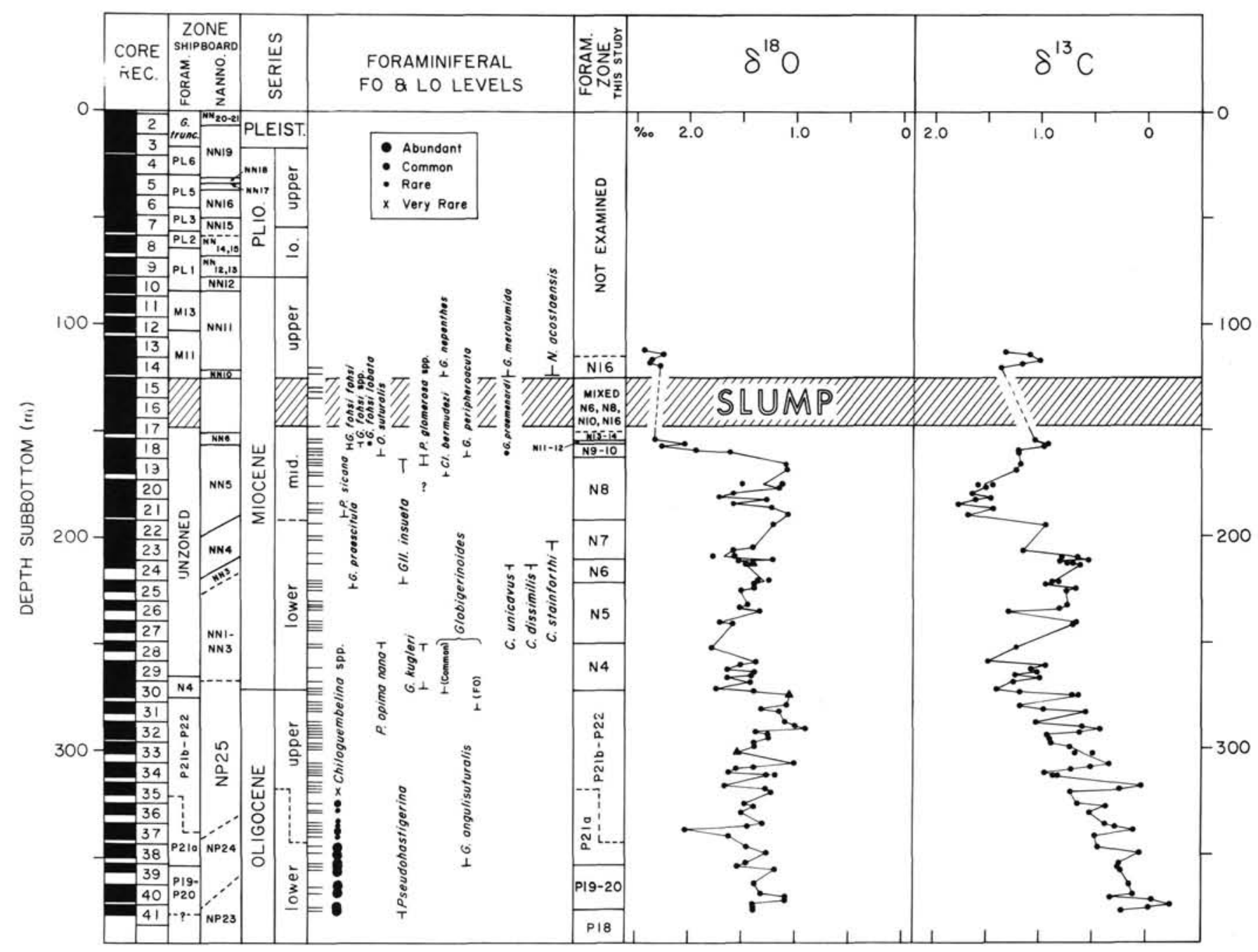

Figure 5. Biostratigraphy and stable isotope stratigraphy, Site 667. REC $=$ recovery. Shading indicates recovered intervals. FO $=$ first occurrences indicated with inverted T's; $\mathrm{LO}=$ last occurrences indicated with T's. Tick marks next to series indicate samples examined for biostratigraphy. Triangles indicate duplicate isotope analyses with the same values. Duplicate isotope analyses are plotted, and a line is drawn through the mean value of the duplicates. Shipboard biostratigraphic zonations are taken from Figure 5 of the site report.

Site 366 (Fig. 4). The mean sedimentation rate for this section is approximately $16 \mathrm{~m} / \mathrm{m} . \mathrm{y} .(1.6 \mathrm{~cm} / \mathrm{k} . \mathrm{y}$.). The zonal markers for the top of Zones P18, P19, and P20 (LO Pseudohastigerina spp., LO "Turborotalia" ampliapertura, and FO Globigerina angulisuturalis, respectively) are well represented. An inter$\mathrm{val}$ of strong dissolution occurs near the base of Hole $366 \mathrm{~A}$ at 348-364 meters below seafloor (mbsf) in the upper part of Zone P19 to the lower part of Zone P20.

The FO of Paragloborotalia opima opima at Site 366 occurs below the FO of $G$. angulisuturalis; five-chambered forms assigned to $P$. cf. $P$. opima opima appear here immediately above the LO of Pseudohastigerina spp. (see "Taxonomic and Stratigraphic Notes," this chapter). At subtropical North Atlantic Site 563, the FO of P. opima opima s.s. $(>390 \mu \mathrm{m})$ occurs immediately above the LO of Pseudohastigerina spp. (Miller et al., 1985b). This effectively eliminates the utility of Bolli's (1957) " $T$." ampliapertura Zone (see Berggren and Miller, 1988; "Taxonomic and Stratigraphic Notes,', this chapter).

We recognize the LO of both Chiloguembelina spp. and $P$. opima opima in the middle of Core 41-366A-33; thus, the lower upper Oligocene Zone P21b is largely missing. The upper Oligocene is approximately $50 \mathrm{~m}$ thick. If the inferred hiatus for Zone P2lb represents the interval from $\sim 29.6 \mathrm{Ma}$ to $28.2 \mathrm{Ma}$, then the mean sedimentation rate for the remaining upper Oligocene section is $\sim 11 \mathrm{~m} / \mathrm{m}$.y. The FO of Globigerinoides spp. (rare) is between Cores 41-366A-31 and -32; the FO of Globorotalia kugleri s.s., which is used to recognize the Oligocene/Miocene boundary (Berggren et al., 1985a, 1985b; see "Taxonomic and Stratigraphic Notes," this chapter), is within Core 41-366A-28 (Fig. 4). Thus, we observe that Globigerinoides appears over $30 \mathrm{~m}(>2.5 \mathrm{~m}$.y.) below the FO of G. kugleri s.s. (see also Lamb and Stainforth, 1976; Berggren et al., 1985a, 1985b; Miller et al., 1985b).

Abundant Globigerinoides appear at the same level as the FO of $G$. kugleri s.s. at Hole $366 \mathrm{~A}$ (Fig. 4). In some sections, the FO of abundant Globigerinoides predates the FO of $G$. kugleri s.s. by about 0.8 m.y. (Berggren et al., 1985a, 1985b). Considering the consistent relationship between diagnostic stable isotope events and the FO of G. kugleri s.s., we suggest that the FO of this taxon is synchronous among locations, while the FO of abundant Globigerinoides is slightly diachronous (see "Taxonomic and Stratigraphic Notes," this chapter). 
Table 1. Oxygen and carbon isotope data, Cibicidoides spp., Site 366.

\begin{tabular}{|c|c|c|c|c|}
\hline $\begin{array}{l}\text { Core, section, } \\
\text { interval }(\mathrm{cm})\end{array}$ & $\begin{array}{l}\text { Depth } \\
\text { (mbsf) }\end{array}$ & $\begin{array}{l}\text { Age } \\
(\mathrm{Ma})\end{array}$ & $\delta^{18} \mathrm{O}$ & $\delta^{13} \mathrm{C}$ \\
\hline \multicolumn{5}{|l|}{$41-366 \mathrm{~A}-$} \\
\hline $13-3,118-121$ & 114.68 & 8.15 & 2.272 & 0.724 \\
\hline $13-5,33-38$ & 116.83 & 8.41 & 2.313 & 0.639 \\
\hline $14-1,11-15$ & 120.11 & 8.81 & 2.265 & 1.142 \\
\hline $14-1,11-15$ & 120.11 & 8.81 & 2.309 & 1.048 \\
\hline $14-3,16-20$ & 123.16 & 9.18 & 2.294 & 0.699 \\
\hline $14-5,26-30$ & 126.26 & 9.55 & 2.121 & 0.815 \\
\hline $15-1,127-130$ & 130.77 & 10.10 & 2.013 & 0.567 \\
\hline $15-3,122-125$ & 133.72 & 11.82 & 2.114 & 0.575 \\
\hline $15-5,134-137$ & 136.84 & 12.59 & 2.131 & 0.537 \\
\hline $16-2,43-47$ & 140.93 & 13.61 & 2.121 & 0.955 \\
\hline $16-3,96-100$ & 142.96 & 14.12 & 1.816 & 1.029 \\
\hline $16-3,96-100$ & 142.96 & 14.12 & 1.864 & 1.174 \\
\hline $16-4,112-116$ & 143.62 & 14.29 & 1.484 & 1.104 \\
\hline $16-5,131-135$ & 146.31 & 16.50 & 1.260 & 1.285 \\
\hline $17-0,32-36$ & 148.82 & 16.66 & 1.981 & 0.855 \\
\hline $17-3,128-132$ & 152.78 & 16.91 & 1.544 & 1.178 \\
\hline $17-5,63-66$ & 155.13 & 17.06 & 1.103 & 1.307 \\
\hline $18-1,123-127$ & 159.23 & 17.31 & 1.536 & 1.765 \\
\hline $18-3,124-128$ & 162.24 & 17.50 & 1.150 & 1.390 \\
\hline $18-5,128-132$ & 165.28 & 17.69 & 1.397 & 1.205 \\
\hline $20-1,111-115$ & 178.11 & 18.49 & 1.527 & 1.281 \\
\hline $20-3,131-135$ & 181.31 & 18.69 & 1.403 & 0.312 \\
\hline $21-2,105-109$ & 189.05 & 19.18 & 1.498 & 0.739 \\
\hline $21-4,128-131$ & 192.28 & 19.38 & 1.373 & 0.570 \\
\hline $21-6,116-120$ & 195.16 & 19.56 & 1.651 & 0.360 \\
\hline $22-2,56-60$ & 198.06 & 19.74 & 1.901 & 0.339 \\
\hline $23-1,10-14$ & 206.73 & 20.28 & 1.671 & 0.481 \\
\hline $23-2,8-11$ & 207.08 & 20.30 & 1.472 & 0.642 \\
\hline $23-3,85-89$ & 209.35 & 20.45 & 1.566 & 0.408 \\
\hline $23-4,127-130$ & 211.27 & 20.57 & 1.512 & 0.814 \\
\hline $23-5,93-97$ & 212.43 & 20.64 & 1.817 & 0.818 \\
\hline $23-6,34-37$ & 213.24 & 20.70 & 1.805 & 0.968 \\
\hline $24-1,142-146$ & 216.42 & 20.89 & 1.502 & 0.756 \\
\hline $24-2,136-139$ & 217.86 & 20.98 & 1.557 & 0.685 \\
\hline $25-1,68-71$ & 225.18 & 21.44 & 1.788 & 0.781 \\
\hline $26-2,140-144$ & 236.90 & 22.21 & 1.711 & 1.056 \\
\hline $26-3,139-143$ & 238.39 & 22.31 & 1.608 & 1.184 \\
\hline $26-4,124-127$ & 239.74 & 22.40 & 1.796 & 1.089 \\
\hline $26-5,125-129$ & 241.25 & 22.51 & 1.630 & 1.258 \\
\hline $27-1,133-137$ & 244.83 & 22.75 & 1.356 & 1.364 \\
\hline $27-2,136-140$ & 246.36 & 22.86 & 1.541 & 1.444 \\
\hline $27-3,143-147$ & 247.93 & 23.97 & 1.285 & 1.310 \\
\hline $27-4,134-138$ & 249.34 & 23.06 & 1.319 & 1.215 \\
\hline $27-4,134-138$ & 249.34 & 23.06 & 1.227 & 1.120 \\
\hline $27-5,140-144$ & 250.90 & 23.17 & 1.227 & 0.840 \\
\hline $27-6,95-99$ & 251.95 & 23.24 & 1.486 & 1.000 \\
\hline $28-1,140-144$ & 254.40 & 23.41 & 1.842 & 1.342 \\
\hline $28-2,89-93$ & 255.39 & 23.48 & 1.710 & 1.420 \\
\hline $28-4,136-140$ & 258.86 & 23.80 & 1.437 & 0.869 \\
\hline $28-5,118-121$ & 260.18 & 23.96 & 1.450 & 0.980 \\
\hline $29-1,136-140$ & 263.86 & 24.38 & 1.259 & 0.590 \\
\hline $29-2,135-139$ & 265.35 & 24.55 & 1.078 & 0.731 \\
\hline $29-2,135-139$ & 265.35 & 24.55 & 1.192 & 0.700 \\
\hline $30-5,96-100$ & 278.91 & 26.12 & 1.230 & 0.650 \\
\hline $31-4,85-89$ & 286.85 & 27.03 & 1.160 & 0.470 \\
\hline $32-\mathrm{CC}$ & 291.00 & 27.51 & 1.170 & 0.740 \\
\hline $33-2,91-95$ & 302.91 & 29.89 & 1.480 & 0.540 \\
\hline $33-2,91-95$ & 302.91 & 29.89 & 1.260 & 0.700 \\
\hline$a_{33-3}, 32$ & 303.82 & 29.94 & 1.790 & 0.930 \\
\hline$a_{33-3,48}$ & 303.98 & 29.94 & 1.840 & 0.910 \\
\hline $33-4,81-85$ & 305.81 & 30.03 & 1.720 & 0.890 \\
\hline $33-5,108-112$ & 307.58 & 30.12 & 1.840 & 0.790 \\
\hline $33-6,81-85$ & 308.81 & 30.18 & 1.470 & 0.700 \\
\hline $34-3,103-107$ & 314.03 & 30.44 & 1.470 & 0.450 \\
\hline $34-5,91-95$ & 316.91 & 30.58 & 1.450 & 0.630 \\
\hline $34-6,103-107$ & 318.53 & 30.66 & 1.575 & 0.276 \\
\hline $34-6,103-107$ & 318.53 & 30.66 & 1.546 & 0.518 \\
\hline $35-2,30-34$ & 321.30 & 30.80 & 1.800 & 0.575 \\
\hline $35-3,40-44$ & 322.90 & 30.88 & 1.360 & 0.010 \\
\hline $35-3,40-44$ & 322.90 & 30.88 & 1.540 & 0.230 \\
\hline $35-6,84-88$ & 327.84 & 31.12 & 1.694 & 0.457 \\
\hline $36-2,84-88$ & 330.90 & 31.27 & 1.490 & 0.550 \\
\hline $36-4,83-87$ & 334.33 & 31.44 & 1.125 & 0.060 \\
\hline $36-5,83-87$ & 335.83 & 31.51 & 1.466 & 0.335 \\
\hline
\end{tabular}

Table 1 (continued).

\begin{tabular}{lllll}
\hline $\begin{array}{c}\text { Core, section, } \\
\text { interval (cm) }\end{array}$ & $\begin{array}{c}\text { Depth } \\
\text { (mbsf) }\end{array}$ & $\begin{array}{c}\text { Age } \\
\text { (Ma) }\end{array}$ & $\delta^{18} \mathrm{O}$ & $\delta^{13} \mathrm{C}$ \\
\hline $41-366 \mathrm{~A}-$ & & & & \\
& & & & \\
$37-1,87-91$ & 339.37 & 31.72 & 1.353 & 0.551 \\
$37-1,87-91$ & 339.37 & 31.72 & 1.180 & 0.450 \\
$37-2,81-85$ & 340.81 & 31.81 & 1.413 & 0.449 \\
$37-6,107-111$ & 347.07 & 32.22 & 1.529 & 0.568 \\
$38-1,105-109$ & 349.05 & 32.35 & 1.645 & 0.306 \\
$38-2,108-111$ & 350.58 & 32.45 & 1.166 & 0.094 \\
$39-1,47$ & 357.97 & 32.89 & 1.310 & 0.070 \\
$39-1,97-100$ & 358.47 & 32.91 & 1.424 & 0.393 \\
$39-1,97-100$ & 358.47 & 32.91 & 1.167 & 0.475 \\
$39-2,88-92$ & 359.88 & 32.98 & 1.314 & 0.243 \\
$39-3,82-85$ & 361.32 & 33.04 & 1.398 & 0.615 \\
$39-4,101-105$ & 363.01 & 33.07 & 1.450 & 0.592 \\
& & & & \\
$41-366-$ & & & & \\
& & & & \\
$5-1,70-74$ & 366.70 & 33.29 & 1.336 & 0.969 \\
$5-2,77-81$ & 368.27 & 33.36 & 0.957 & 0.767 \\
$5-2,77-81$ & 368.27 & 33.36 & 1.106 & 0.538 \\
$5-3,70-74$ & 369.70 & 33.42 & 1.372 & 1.006 \\
$5-4,70-74$ & 371.20 & 33.49 & 1.134 & 0.678 \\
$5-5,64-68$ & 372.64 & 33.56 & 1.126 & 0.661 \\
$5-6,73-77$ & 374.23 & 33.63 & 1.446 & 0.633 \\
$6-1,110-114$ & 376.60 & 33.74 & 1.071 & 0.548 \\
$6-2,105-109$ & 378.05 & 33.80 & 1.254 & 0.898 \\
$6-4,96-100$ & 380.96 & 33.94 & 1.654 & 0.850 \\
$6-6,95-99$ & 383.95 & 34.14 & 1.209 & 0.924 \\
$7-2,95-99$ & 387.45 & 34.45 & 1.377 & 0.786 \\
$7-3,102-106$ & 389.02 & 34.58 & 1.130 & 0.606 \\
$8-1,139-143$ & 395.89 & 35.18 & 1.347 & 1.052 \\
$8-1,139-143$ & 395.89 & 35.18 & 1.467 & 1.026 \\
$8-3,103-107$ & 398.53 & 35.42 & 1.110 & 0.828 \\
$9-1,93-97$ & 404.93 & 35.98 & 1.379 & 1.266 \\
$9-4,105-109$ & 409.55 & 36.38 & 0.551 & 1.018 \\
$10-2,38-42$ & 415.38 & 36.89 & 0.610 & 0.780 \\
$10-5,74-78$ & 420.24 & 37.31 & 0.350 & 0.579 \\
$11-1,119-123$ & 424.19 & 37.66 & 0.395 & 1.181 \\
$12-1,96-100$ & 433.46 & 38.47 & 0.323 & 1.133 \\
\hline & & & & \\
\hline & & & & \\
\hline
\end{tabular}

${ }^{a}$ Analysis after Boersma and Shackleton, 1978.

Note: Ages have been estimated to two decimal places to reflect the relative position of closely spaces samples (e.g., Samples 41-366A-39-1, $47 \mathrm{~cm}$, and $41-366 \mathrm{~A}-39-1,97 \mathrm{~cm}$ ) and do not reflect accuracy of correlations.

At Hole 366A, the lower Miocene is unconformably overlain by the middle Miocene, which is unconformably overlain by the upper Miocene (Krasheninnikov and Pflaumann, 1978b). We observe the LO of Catapsydrax dissimilis and $C$. unicavus ( $\sim 17.6 \mathrm{Ma}$; Berggren et al., 1985b) at the base of Core 41-366A-18; Krasheninnikov and Pflaumann (1978b) observe the LO of this taxon at the top of Core 41-366A-20. This discrepancy is minor considering that there was no recovery from Core 41-366A-19. Krasheninnikov and Pflaumann (1978b) report the absence of the Praeorbulina glomerosa Zone and the Orbulina suturalis-Globorotalia peripheroronda Zone ( $\sim$ Zones N8-N9) and suggest therefore that the lower/middle Miocene contact is disconformable. There is approximately $108 \mathrm{~m}$ of lower Miocene section at Hole 366A, with a mean sedimentation rate of approximately $15 \mathrm{~m} / \mathrm{m} . \mathrm{y}$.

The middle Miocene is poorly represented at Hole $366 \mathrm{~A}$, being only about 15-16 m thick (Krasheninnikov and Pflaumann, 1978b). In order to estimate ages from this section, we assumed that the FO of Globorotalia peripheroacuta at Hole $366 \mathrm{~A}$ was a true evolutionary $\mathrm{FO}$, and that the LO of G. fohsi and $G$. fohsi lobata-robusta were evolutionary extinctions (datum levels picked by Krasheninnikov and Pflaumann, 1978b). The FO of Orbulina in this section is interpreted as 
Table 2. Oxygen and carbon isotope data, Cibicidoides spp., Site 667.

\begin{tabular}{|c|c|c|c|c|}
\hline $\begin{array}{l}\text { Core, section, } \\
\text { interval }(\mathrm{cm})\end{array}$ & $\begin{array}{l}\text { Depth } \\
\text { (mbsf) }\end{array}$ & $\begin{array}{l}\text { Age } \\
\text { (Ma) }\end{array}$ & $\delta^{18} \mathrm{O}$ & $\delta^{13} \mathrm{C}$ \\
\hline \multicolumn{5}{|l|}{$108-667 \mathrm{~A}-$} \\
\hline $25 \mathrm{X}-1,36-40$ & 220.16 & 18.79 & 1.349 & 0.884 \\
\hline $25 X-1,36-40$ & 220.16 & 18.79 & 1.254 & 0.832 \\
\hline $25 X-2,36-40$ & 221.66 & 18.96 & 1.383 & 0.954 \\
\hline $25 \mathrm{X}-3,36-40$ & 223.16 & 19.12 & 1.382 & 0.673 \\
\hline $25 X-4,42-46$ & 224.72 & 19.30 & 1.510 & 0.762 \\
\hline $26 \mathrm{X}-2,36-40$ & 231.16 & 20.03 & 1.445 & 0.738 \\
\hline $26 \mathrm{X}-3,36-40$ & 232.66 & 20.19 & 1.521 & 0.811 \\
\hline $26 \mathrm{X}-4,36-40$ & 234.16 & 20.36 & 1.336 & 1.290 \\
\hline $27 \mathrm{X}-1,36-40$ & 239.16 & 20.93 & 1.711 & 0.671 \\
\hline $27 X-2,36-40$ & 240.66 & 21.10 & 1.509 & 0.701 \\
\hline $28 \mathrm{X}-3,36-40$ & 251.66 & 22.17 & 1.786 & 1.234 \\
\hline $29 \mathrm{X}-1,36-39$ & 258.16 & 22.67 & 1.367 & 1.502 \\
\hline $29 \times-2,36-39$ & 259.66 & 22.79 & 1.524 & 0.954 \\
\hline $29 \mathrm{X}-3,39-42$ & 261.19 & 22.90 & 1.650 & 1.086 \\
\hline $29 \times-4,36-39$ & 262.66 & 23.02 & 1.390 & 1.042 \\
\hline $29 X-5,36-39$ & 264.16 & 23.13 & 1.424 & 1.245 \\
\hline $29 X-6,40-43$ & 265.70 & 23.25 & 1.646 & 1.013 \\
\hline $30 \mathrm{X}-1,36-39$ & 267.66 & 23.40 & 1.431 & 1.261 \\
\hline $30 X-3,50-53$ & 270.80 & 23.65 & 1.739 & 1.399 \\
\hline $30 \times-4,36-39$ & 272.16 & 23.76 & 1.393 & 1.179 \\
\hline $30 \times-5,36-39$ & 273.66 & 23.89 & 1.050 & 0.623 \\
\hline $30 \times-5,36-39$ & 273.66 & 23.89 & 1.053 & 0.693 \\
\hline $31 \times-2,36-39$ & 278.66 & 24.34 & 1.080 & 1.178 \\
\hline $31 \times-3,36-39$ & 280.16 & 24.54 & 1.308 & 0.952 \\
\hline $31 X-4,41-44$ & 281.66 & 24.47 & 1.153 & 0.549 \\
\hline $32 X-1,30-33$ & 286.60 & 25.04 & 1.088 & 1.034 \\
\hline $32 \times-2,36-39$ & 288.16 & 25.18 & 1.007 & 0.596 \\
\hline $32 \times-3,36-39$ & 289.66 & 25.31 & 0.904 & 0.425 \\
\hline $32 \times-4,36-39$ & 291.16 & 25.44 & 1.372 & 0.626 \\
\hline $32 \times-5,36-39$ & 292.66 & 25.57 & 1.260 & 0.934 \\
\hline $32 \times-6,36-39$ & 294.16 & 25.71 & 1.251 & 0.907 \\
\hline $33 \mathrm{X}-1,84-88$ & 296.64 & 25.93 & 1.391 & 0.879 \\
\hline $33 X-2,87-91$ & 298.17 & 26.06 & 1.385 & 0.709 \\
\hline $33 X-4,88-92$ & 301.18 & 26.33 & 1.530 & 0.486 \\
\hline $33 X-4,88-92$ & 301.18 & 26.33 & 1.537 & 0.657 \\
\hline $34 \mathrm{X}-1,66-69$ & 305.96 & 26.75 & 1.010 & 0.330 \\
\hline $34 X-2,65-68$ & 307.45 & 26.88 & 1.377 & 0.514 \\
\hline $34 X-3,84-87$ & 309.14 & 27.03 & 1.558 & 0.699 \\
\hline $34 X-4,76-78$ & 310.56 & 27.16 & 1.624 & 0.943 \\
\hline $34 X-5,11-13$ & 311.41 & 27.23 & 1.268 & 0.823 \\
\hline $34 X-5,11-13$ & 311.41 & 27.23 & 1.188 & 0.859 \\
\hline $35 X-2,25-29$ & 316.55 & 27.69 & 1.661 & 0.035 \\
\hline $35 X-3,35-39$ & 318.15 & 27.83 & 1.281 & 0.244 \\
\hline $35 X-4,35-39$ & 319.65 & 27.96 & 1.235 & 0.704 \\
\hline $36 \times-1,60-64$ & 324.90 & 28.43 & 1.472 & 0.631 \\
\hline $36 \times-2,26-29$ & 326.06 & 28.53 & 1.312 & 0.367 \\
\hline $36 \mathrm{X}-4,22-25$ & 329.02 & 28.79 & 1.505 & 0.520 \\
\hline $37 X-1,38-41$ & 334.18 & 29.25 & 1.298 & 0.384 \\
\hline $37 X-2,42-44$ & 335.64 & 29.38 & 1.446 & 0.277 \\
\hline $37 X-3,35-38$ & 337.15 & 29.51 & 2.019 & 0.109 \\
\hline $37 X-5,36-39$ & 340.16 & 29.78 & 1.618 & 0.468 \\
\hline $38 \mathrm{X}-2,39-42$ & 345.19 & 30.36 & 1.445 & 0.443 \\
\hline $38 \times-4,36-39$ & 348.16 & 30.78 & 1.274 & 0.052 \\
\hline $39 \mathrm{X}-1,36-39$ & 353.16 & 31.49 & 1.458 & 0.238 \\
\hline $39 \mathrm{X}-2,36-39$ & 354.66 & 31.69 & 1.545 & 0.252 \\
\hline $39 \times-3,36-40$ & 356.16 & 31.86 & 1.190 & 0.226 \\
\hline $40 X-1,30-34$ & 362.60 & 32.60 & 1.381 & 0.148 \\
\hline $40 X-4,30-34$ & 367.10 & 32.12 & 1.317 & 0.109 \\
\hline $40 X-5,30-34$ & 368.60 & 33.29 & 1.091 & 0.323 \\
\hline $40 X-6,30-34$ & 370.10 & 33.47 & 1.093 & -0.064 \\
\hline $41 X-1,36-40$ & 372.16 & 33.70 & 1.392 & -0.235 \\
\hline $41 X-2,36-40$ & 373.66 & 33.88 & 1.390 & -0.042 \\
\hline $41 X-3,36-40$ & 375.16 & 34.05 & 1.386 & 0.221 \\
\hline
\end{tabular}

Note. Ages have been estimated to two decimal places to reflect the relative position of closely spaced samples (e.g., Samples 41-366A-39-1, $47 \mathrm{~cm}$, and $41-366 \mathrm{~A}-39-1,97 \mathrm{~cm}$ ) and do not reflect accuracy of correlations.
Table 3. Site 366 biostratigraphic datum levels.

\begin{tabular}{|c|c|c|}
\hline Criterion & $\begin{array}{l}\text { Depth } \\
\text { (mbsf) }\end{array}$ & $\begin{array}{l}\text { Age } \\
\text { (Ma) }\end{array}$ \\
\hline FO Amaurolithus primus ${ }^{\mathrm{a}}$ & 101.00 & 6.5 \\
\hline $\begin{array}{l}\text { FO Neogloboquadrina } \\
\text { acostaensis }\end{array}$ & 131.61 & 10.2 \\
\hline Unconformity & 131.62 & $11.3-10.2$ \\
\hline FO Globorotalia fohsi robusta ${ }^{b}$ & 136.88 & 12.6 \\
\hline FO Globorotalia peripheroacuta ${ }^{b}$ & 146.07 & 14.9 \\
\hline Unconformity & 146.08 & $16.5-14.9$ \\
\hline LO Catapsydrax dissimilis & 163.85 & 17.6 \\
\hline LO Globorotalia kugleri & 231.00 & 21.8 \\
\hline FO Globorotalia kugleri & 257.10 & 23.6 \\
\hline LO Paragloborotalia-opima opima & 296.96 & 28.2 \\
\hline Unconformity & 296.97 & $29.6-28.2$ \\
\hline LO Chiloguembelina spp. & 305.13 & 30.0 \\
\hline FO Globigerina angulisuturalis & 337.60 & 31.6 \\
\hline LO Globigerina ampliapertura & 356.01 & 32.8 \\
\hline LO Pseudohastigerina spp. & 382.34 & 34.0 \\
\hline LO Hantkenina spp. & 412.07 & 36.6 \\
\hline
\end{tabular}

Bukry, 1978

b Krasheninnikov and Pflaumann, 1978a.

delayed, for it first appears above the $\mathrm{FO}$ of G. peripheroacuta at Hole 366A (Fig. 4; Krasheninnikov and Pflaumann, 1978b), while elsewhere it first occurs below this FO (Berggren et al., 1985b).

The contact between the middle and upper Miocene at Hole $366 \mathrm{~A}$ is interpreted as disconformable, for the $\mathrm{LO}$ of $G$. fohsi spp. is at the same level as the LO of G. mayerisiakensis and immediately below the FO of Neogloboquadrina acostaensis (Fig. 4; Krasheninnikov and Pflaumann, 1978b). At least 1.3 m.y. are missing across this boundary. Our study did not extend into the upper upper Miocene (Messinian equivalent); our uppermost samples from Core 41-366A-13 are older than $8 \mathrm{Ma}$ (Tortonian equivalent), well below the $\mathrm{FO}$ of Amaurolithus primus at approximately $101 \mathrm{~m}$ (between unspecified intervals in Sections 41-366A-11-3 and 41-366A-12-3; Bukry, 1978).

\section{Site 667}

Hole 667 bottomed in the lower Oligocene at $381 \mathrm{mbsf}$ in Zone P18 (Fig. 5). Rare Pseudohastigerina occurs in the lowermost sample examined (108-667A-41X-3, 36-40 cm), indicating that the hole bottomed in strata slightly older than $34 \mathrm{Ma}$. The distribution of "Turborotalia" ampliapertura is sporadic at this hole due to poor preservation in the lower Oligocene section; therefore, we cannot distinguish between Zones P19 and P20 at this borehole. The rarity of Paragloborotalia opima opima may be similarly attributed to poor preservation. Globigerina angulisuturalis first appears near the top of Core 108-667A-39X (Fig. 5), marking the base of Zone P21a. The LO of Chiloguembelina is in the interval of Cores 108-667A-35X to -37X: it becomes rare at the base of Core 108-667A-37X, straggling up to the top of Core 108667A-35X (Fig. 5).

The P21a/P21b zonal boundary can be placed at the base of this interval assuming reworking, a hypothesis that we favor; alternatively, the zonal boundary and the associated lower/ middle Oligocene boundary may be placed at the top of the interval if we assume that the rare specimens are in situ. Zones P21b and P22 are undifferentiated due to the scarcity of $P$. opima opima, and a hiatus such as noted for Zone P2lb at Hole $366 \mathrm{~A}$ may or may not be present. There are approximately $110 \mathrm{~m}$ of Oligocene strata at Hole 667 , with a mean sedimentation rate of about $10 \mathrm{~m} / \mathrm{m}$.y. (assuming no hiatuses). Assuming that the upper/lower Oligocene boundary is at the 
Table 4. Site 667 biostratigraphic datum levels.

\begin{tabular}{lrr}
\hline \multicolumn{1}{c}{ Criterion } & $\begin{array}{r}\text { Depth } \\
\text { (mbsf) }\end{array}$ & $\begin{array}{r}\text { Age } \\
\text { (Ma) }\end{array}$ \\
\hline Zone NN11/NN12 boundary & 84.16 & 5.6 \\
$\begin{array}{l}\text { Zone NN10/NN11 boundary } \\
\text { Extrapolation of sedimentation }\end{array}$ & 120.55 & 8.2 \\
$\quad$ rate from above & 125.00 & 8.5 \\
Zone N13/N14 & 153.65 & 11.3 \\
Globorotalia fohsi lobata & 155.16 & 12.9 \\
$\quad$ (13.1-12.7) & 158.16 & 13.5 \\
Middle of Zone N11 & 159.66 & 14.4 \\
Middle of Zone N10 & 161.16 & 14.9 \\
FO Globorotalia & & \\
$\quad$ peripheroacuta & 163.16 & 15.2 \\
FO Orbulina spp. & 209.63 & 17.6 \\
LO Catapsydrax dissimilis & 246.91 & 21.8 \\
LO Globorotalia kugleri & 271.48 & 23.7 \\
FO Globorotalia kugleri & 342.68 & 30.0 \\
LO Chiloguembelina spp. & 353.91 & 31.6 \\
FO Globigerina angulisuturalis & 374.74 & 34.0 \\
\hline LO Pseudohastigerina spp. & & \\
\hline
\end{tabular}

Table 5. Site 366 tuned-age model.

\begin{tabular}{lcc}
\hline \multicolumn{1}{c}{ Criterion } & $\begin{array}{c}\text { Depth } \\
\text { (mbsf) }\end{array}$ & $\begin{array}{c}\text { Age } \\
\text { (Ma) }\end{array}$ \\
\hline Equivalent to a level of: & \\
& & \\
$159.7 \mathrm{~m}$ at Site 563 & 114.68 & 8.2 \\
$185.4 \mathrm{~m}$ at Site 563 & 123.61 & 10.2 \\
Unconformity & 131.62 & $11.7-10.2$ \\
$230.1 \mathrm{~m}$ at Site 563 & 140.93 & 13.1 \\
$235.2 \mathrm{~m}$ at Site 563 & 143.62 & 13.5 \\
Unconformity & 146.08 & $14.9-13.9$ \\
$254.0 \mathrm{~m}$ at Site 563 & 162.24 & 16.6 \\
$273.2 \mathrm{~m}$ at Site 563 & 192.28 & 19.8 \\
$298.0 \mathrm{~m}$ at Site 563 & 250.91 & 23.4 \\
$307.0 \mathrm{~m}$ at Site 563 & 265.35 & 25.0 \\
\hline
\end{tabular}

top of Core $108-667 \mathrm{~A}-38 \mathrm{X}$, there is about $72 \mathrm{~m}$ of upper Oligocene and $38 \mathrm{~m}$ of lower Oligocene.

The base of the Miocene is well marked by the FO of Globorotalia kugleri within Core 108-667A-30X (Fig. 5). Globigerinoides first appears in the middle of Core 108-667A$31 \mathrm{X}$, and it appears in abundance at the base of Core 108667A-30X. Globorotalia kugleri last appears at the top of Core 108-667A-28X, marking the top of Zone N4. Paragloborotalia opima nana also disappears at this level. We have noted the persistence of this purported Oligocene taxon into Miocene Zone N4 at North Atlantic subtropical Site 563, and we suggest that the LO of this taxon may prove useful in recognizing basal Miocene strata (see "Taxonomic and Stratigraphic Notes," this chapter).

Globigerinatella insueta appears in the middle of Core 108-667A-25X, marking the top of Zone N5 as defined. Because this taxon is often rare or absent in many sections (particularly in the early part of its range), and because Zone N5 is a "gap zone," we suggest that a consistent temporal correlation of Zone N5 is not possible even among tropical or subtropical locations (see "Taxonomic and Stratigraphic Notes," this chapter). For example, we have been unable to differentiate Zone N5 from Zone N6 due to the absence of $G$. insueta at Sites 366, 558, and 563 (Miller et al., 1985a; this study). Catapsydrax dissimilis and $C$. unicavus disappear at the top of Core 108-667A-24X, marking the top of Zone N6. Praeorbulina sicana first appears in Core 108-667A-21H, indicating the base of Zone N8 and the middle Miocene.
There are about $80 \mathrm{~m}$ of lower Miocene strata at Hole 667 with a mean sedimentation rate of about $11 \mathrm{~m} / \mathrm{m}$.y. The Oligocene to lower Miocene sedimentation rates at this hole and at Site 366 are relatively constant, ranging from 11-16 $\mathrm{m} / \mathrm{m}$.y. Middle Miocene Zone N8 is well represented at Hole 667, unlike at Hole 366A (Krasheninnikov and Pflaumann, 1978b). The middle Miocene is otherwise condensed at Hole 667 , as it is at Hole $366 \mathrm{~A}$.

Praeorbulina glomerosa first appears near the top of Core 108-667A-19H; Orbulina appears at the base of Core 108$667 \mathrm{~A}-18 \mathrm{H}$, marking the base of undifferentiated Zones N9N10. Globorotalia fohsi fohsi first appears in the middle of Core 108-667A-18H, while Globorotalia fohsi lobata appears only in Section 108-667A-18H-2, 36-41 cm. No G. fohsi spp. are found above this level. Thus, Zones N9 through N12 are condensed into an approximately $10-\mathrm{m}$ interval, while Zones N13-N14 (undifferentiated) are found only above Section $108-667 \mathrm{~A}-18 \mathrm{H}-2,36-41 \mathrm{~cm}$, and below the slump that begins less than $10 \mathrm{~m}$ above this level.

The slump lies between the middle of Core 108-667A-17H and the top of Core 108-667A-15H (Fig. 5). We noted mixed assemblages of various ages in the slumped sediments, including Zones N6, N8, N10, and N16. We estimate that about 3 m.y. are missing in the interval of the slump (Table 3). Globigerina nepenthes first appears immediately above the slump. We did not observe Globorotalia mayeri-siakensis in Core 108-667A-14H, but we did note the presence of Neogloboquadrina acostaensis and Globorotalia merotumida (Fig. 5), which appears at Site 558 with Neogloboquadrina acostaensis (Miller et al., 1985a). We therefore place Core 108$667 \mathrm{~A}-14 \mathrm{H}$ into upper Miocene Zone N16. No samples were examined above Core 108-667A-14H (Fig. 5).

\section{Isotopes}

Oxygen isotope values increase by approximately $0.9 \%$ in the basal Oligocene at Hole 366 (about 36.0 Ma; Table 1), immediately above the LO of the planktonic foraminifer taxa used to recognize the Eocene/Oligocene boundary (Fig. 4; Berggren et al., 1985a). The magnitude of this increase is smaller than observed at other locations at this time (typically $>1 \%$ ) , and $\delta^{18} \mathrm{O}$ values in the lowermost Oligocene $(\sim 1.3 \%$ ) are lower than observed elsewhere (often exceeding 2.0\%; e.g., Leg 74 sites, Shackleton et al., 1984; Site 522, Poore and Matthews, 1984, and Miller et al., 1988; see summary in Miller et al., 1987a). Values similar to those at lowermost Oligocene Hole 366 have been observed in the lowermost Oligocene at Pacific Sites 77 (Keigwin and Keller, 1984) and 574 (Miller and Thomas, 1985), but these records may suffer from slight alteration due to contact with the upper Eocene basement rock, which may have lowered $\delta^{18} \mathrm{O}$ values.

We suspect that the $\delta^{18} \mathrm{O}$ records from the lowermost Oligocene at Hole 366 may be slightly overprinted by diagenesis. There is little optical evidence of diagenetic alteration (crystalline overgrowths or recrystallization); however, two lines of evidence suggest that the lowermost Oligocene $\delta^{18} \mathrm{O}$ records at Hole 366 may be partially altered. First, the lower Oligocene at Hole 366 is well-indurated chalk with a burial depth of approximately $400 \mathrm{~m}$; burial depths greater than $350-400 \mathrm{~m}$ commonly show alteration of the $\delta^{18} \mathrm{O}$ signal (Miller et al., 1987a). Second, most Atlantic isotope records show a characteristic interval of high $\delta^{18} \mathrm{O}$ values $(>2 \%$ in the earliest Oligocene, about $36-35 \mathrm{Ma}$ ), with slightly lower values $(\sim 1.6 \%$ ) from about $34-33 \mathrm{Ma}$. This feature is not observed at Hole 366 (Fig. 4), leading us to suspect a slight diagenetic lowering of lowermost Oligocene $\delta^{18} \mathrm{O}$ values.

High $\delta^{13} \mathrm{C}$ values in the lowermost Oligocene at Hole 366 are followed by a distinct "middle" Oligocene minimum observed at 
both Sites 366 and 667 (Figs. 4 and 5). This $\delta^{13} \mathrm{C}$ minimum is a global phenomenon (Miller and Fairbanks, 1985). High $\delta^{18} \mathrm{O}$ values $(>1.6 \%$ ) occur in the "middle" Oligocene at Site 366 , with several values exceeding $1.8 \%$ (Fig. 4). This "middle" Oligocene interval of high $\delta^{18} \mathrm{O}$ is poorly represented at Hole 667 (Fig. 5), perhaps owing to an undetected unconformity. At both locations, $\delta^{18} \mathrm{O}$ values decrease in the upper Oligocene, but they sharply increase immediately below the Oligocene/Miocene boundary (Figs. 4 and 5).

Carbon isotope values also show a distinct increase immediately below the Oligocene/Miocene boundary. This distinctive increase in $\delta^{18} \mathrm{O}$ and $\delta^{13} \mathrm{C}$ also has been observed at North Atlantic Sites 558 and 563 (Fig. 1; Miller et al., 1985a) and at South Atlantic Site 522 (Miller et al., 1988). Maximum $\delta^{18} \mathrm{O}$ and $\delta^{13} \mathrm{C}$ values at all locations occur at or near the $\mathrm{FO}$ of Globorotalia kugleri s.s. and therefore are useful markers of the Oligocene/Miocene boundary in these pelagic sections.

The lower to middle Miocene at both Sites 366 and 667 show a $\delta^{13} \mathrm{C}$ pattern of decreasing values in Zones N5-N6, followed by a distinct increase in Zones N7-N8 (and possibly in upper Zone N6), and decreasing values in the middle Miocene (Figs. 4 and 5). This pattern has been observed globally (Miller and Fairbanks, 1985; Vincent and Berger, 1985) and reflects the changing input or output ratios of organic carbon to carbonate carbon (Miller and Fairbanks, 1985; Shackleton, 1987).

The lower Miocene $\delta^{18} \mathrm{O}$ records at Sites 366 and 667 show no overall trends (Figs. 4 and 5). Neither site displays the minimum in $\delta^{18} \mathrm{O}$ noted elsewhere (e.g., Pacific Site 289, Woodruff et al., 1981). A distinct $\delta^{18} \mathrm{O}$ increase occurs in the middle Miocene at both locations (Figs. 4 and 5). This increase has been noted globally (e.g., Savin et al., 1975; Shackleton and Kennett, 1975; Woodruff et al., 1981; Miller and Fairbanks, 1985; among others). Our lower upper Miocene records show no overall trends because of limited sample coverage.

The utility of the earliest Oligocene and middle Miocene $\delta^{18} \mathrm{O}$ increases has been widely recognized. The $\delta^{18} \mathrm{O}$ increase associated with the Oligocene/Miocene boundary has been reported at Sites $366,563,558$, and 667 . We suggest that the consistent association of the $\delta^{18} \mathrm{O}$ and $\delta^{13} \mathrm{C}$ increases with the FO of Globorotalia kugleri s.s. (Figs. 4 and 5) argues for its synchrony. As noted above, the FO of Globigerinoides spp. (abundance) is at the $\delta^{18} \mathrm{O}$ and $\delta^{13} \mathrm{C}$ maxima at Site 366, but well below them at Site 667 .

Since the relationship of the FO of Globigerinoides spp. (both FO and first abundant occurrence) vs. stable isotopes is variable from site to site, we suggest that the Globigerinoides abundance "datum level"' is diachronous. The other alternative, that the isotope maxima and associated FO of G. kugleri s.s. are diachronous, is refuted by relationships observed at Sites 563 and 558. At these sites, the isotope maxima and the FO of G. kugleri occur consistently near the Magnetochron $\mathrm{C} 6 \mathrm{Cn} / \mathrm{C} 6 \mathrm{Cr}$ boundary (Miller et al., 1985a), arguing for their synchrony.

\section{DISCUSSION}

Comparisons of biostratigraphically constrained age estimates for stable isotope records from Sites 366 and 667 show good agreement with each other and with records from Site 563 (Figs. 6 and 7); biostratigraphic age estimates are provided

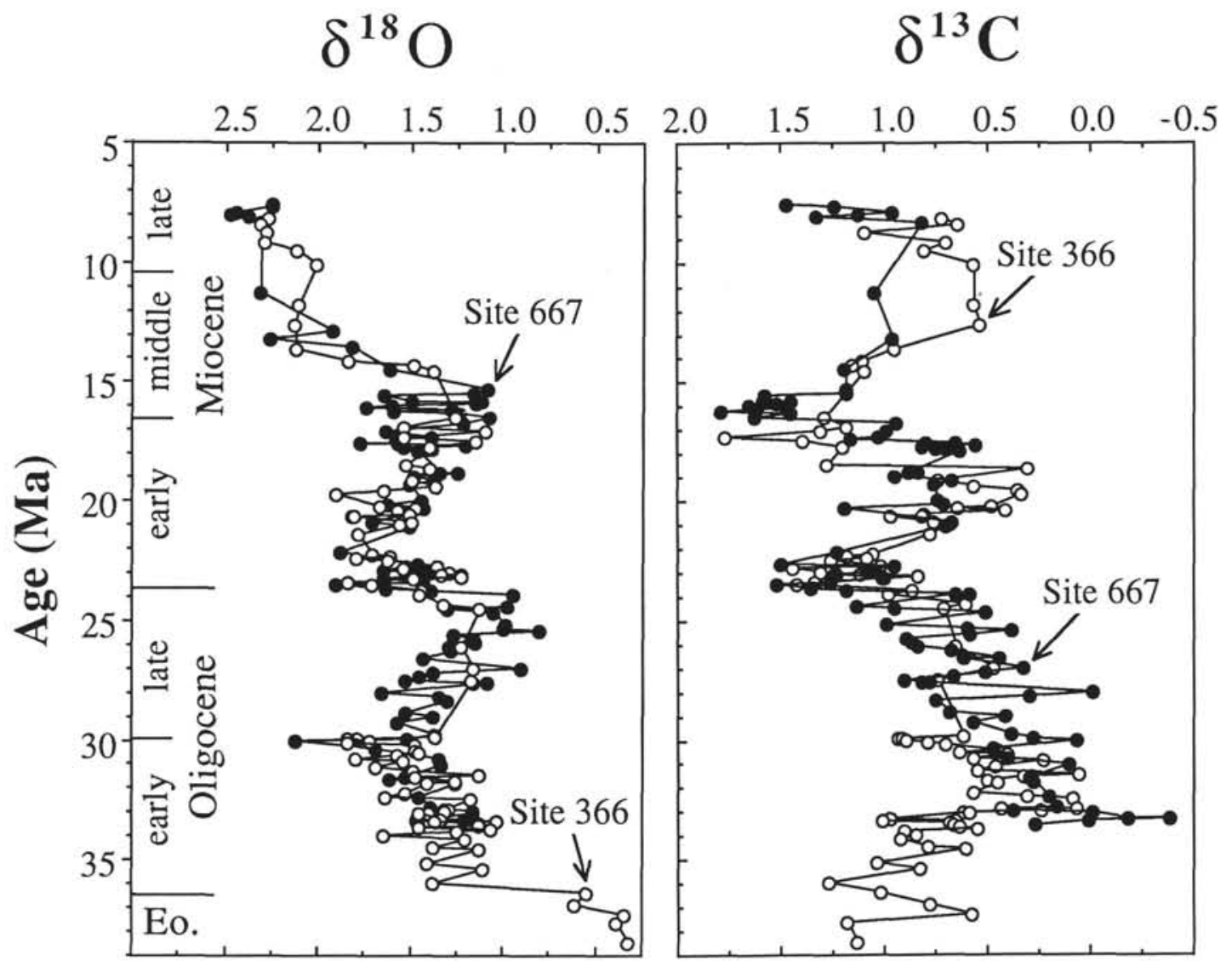

Figure 6. Comparisons of age estimates for Sites 366 (open circles) and 667 (closed circles) using the age models given in Tables 1 through 5 . 


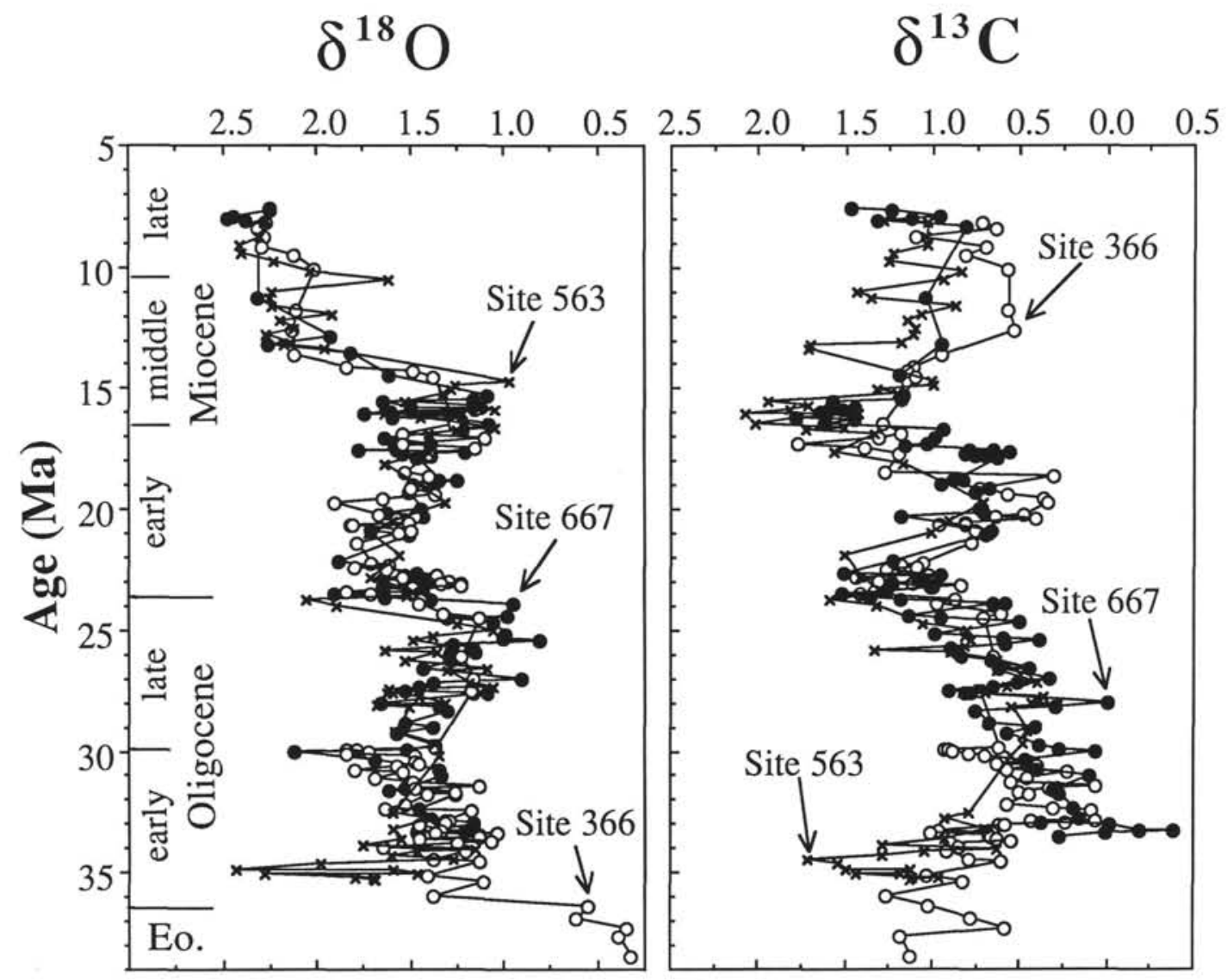

Figure 7. Comparisons of age estimates for Sites 366 (open circles), 667 (closed circles), and western North Atlantic Site 563 ( $x$ 's). The age model for Site 563 is from Miller et al. (1987a).

in Tables 3 and 4 . The general $\delta^{18} \mathrm{O}$ trends are reproduced in both records; in particular, the timing of $\delta^{18} \mathrm{O}$ increases near the Oligocene/Miocene boundary (about 24-23 Ma) and in the middle Miocene (about 15-13 Ma) are similar. The general $\delta^{18} \mathrm{C}$ trends also are reproduced, and the timing of the peak $\delta^{13} \mathrm{C}$ near the Oligocene/Miocene boundary (about 24-23 Ma) is the same at both locations. However, the carbon isotope records show an offset or apparent time lag from about 18 to $16 \mathrm{Ma}$, with the Site 667 record lagging behind the Site 366 record (Fig. 6). We ascribe the apparent lag in the carbon isotope records to stratigraphic problems. Using carbon isotope records to correlate Sites 366 and 667 suggests that

1. a level of about $210 \mathrm{~m}$ at Site 667 (immediately above the LO Catapsydrax dissimilis) is equivalent to a level of about $175 \mathrm{~m}$ at Site 366 (which is below our pick of the LO Catapsydrax dissimilis); and

2. a level of about $180 \mathrm{~m}$ at Site 667 (near the base of Zone N8, above the FO Praeorbulina sicana) is equivalent to a level of about $160 \mathrm{~m}$ at Site 366 (within Zone N7).

These carbon isotope correlations suggest that our pick of the LO of $C$. dissimilis may be too high at Site 366 and that the determination of Krasheninnikov and Pflaumann (1978b) is correct. This is reasonable given the lack of recovery in Core 41-366-19 and the possibility of reworking into the only sample above Core 41-366-19 that contains $C$. dissimilis. The carbon isotope correlations also predict that Praeorbulina sicana should occur at about $160 \mathrm{~m}$ at Site 366 , or that our identification of its FO at Site 667 is incorrect. Such a disagreement among studies is not surprising considering the problems of picking the FO of a taxon in such an evolution- ary bioseries as the Globigerinoides trilobus $-P$. sicana-P. glomerosa lineage.

Oxygen isotope variations provide a means for fine tuning stratigraphic correlations among locations. As an example of this technique, we used the Miocene portion of the Site 563 $\delta^{18} \mathrm{O}$ record as the standard by which we correlated our Site $366 \delta^{18} \mathrm{O}$ record (Fig. 8). We apply the term "tuning" to this correlation method, for we adjust the age of an "unknown" signal to a standard section. The choice of Site 563 as a standard is arbitrary in this case: we could have used the Site 608 section, which contains better magnetostratigraphy for the standard, but the $\delta^{18} \mathrm{O}$ record is not as complete as at Site 563 . The tuned record at Site 366 aligns fine-scale (on the m.y. scale or finer) $\delta^{18} \mathrm{O}$ variations with those at Site 563 (Fig. 8 and Table 5); the realignment on the basis of $\delta^{18} \mathrm{O}$ stratigraphy improves the correlation between the $\delta^{13} \mathrm{C}$ records at Sites 563 and 366 (Fig. 8).

The implications of this oxygen isotope correlation (Fig. 8 and Table 5) include (1) the elimination of the apparent time lag shown on Figure 6 between Sites 366 and 667 between 18 and $16 \mathrm{Ma}$; (2) the prediction that a level of $160 \mathrm{~m}$ at Site 366 is about $16.3 \mathrm{Ma}$, equivalent to a level of about $184 \mathrm{~m}$ at Site 667 , which agrees with predictions from the carbon isotope comparisons; (3) the prediction that the FO of Praeorbulina sicana $(16.6 \mathrm{Ma})$ should be at about $162 \mathrm{~m}$ at Site 366 ; (4) the prediction that a level of $175 \mathrm{~m}$ at Site 366 is $17.6 \mathrm{Ma}$, which agrees with the correlation based upon carbon isotopes; (5) the prediction that the true LO of Catapsydrax dissimilis (17.6 $\mathrm{Ma})$ at Site 366 should be at about $175 \mathrm{~m}$, near the level observed by Krasheninnikov and Pflaumann (1978b; top of 

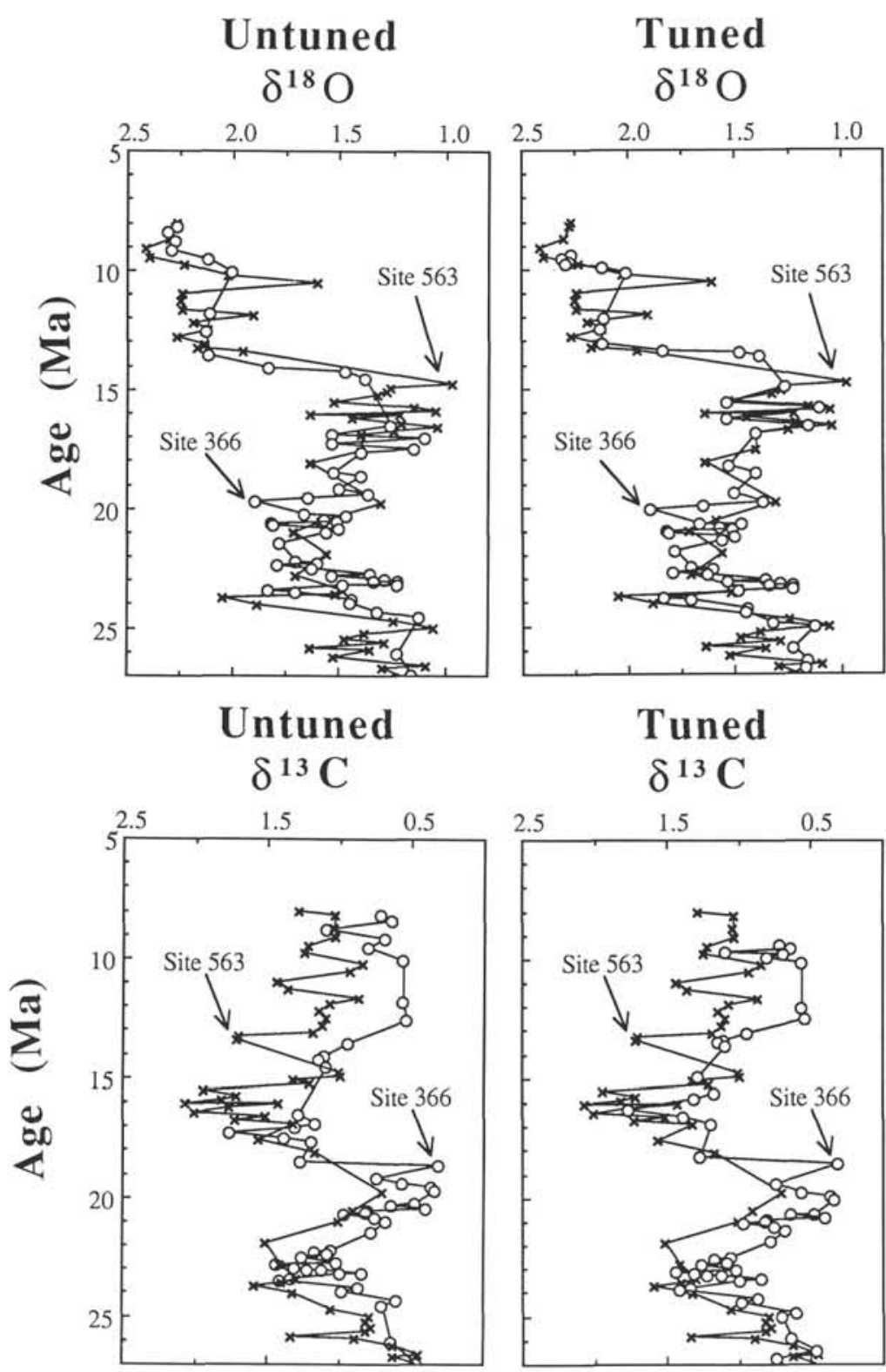

\section{Tuned}

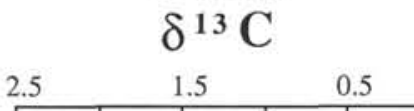

Figure 8. Example of tuning. Comparisons of oxygen and carbon isotope records from western North Atlantic Site 563 and eastern basin Site 366. Untuned-age models use biostratigraphy (Tables 3 and 4 ); tuned-age models assume the correlation of fine-scale $\delta^{18} \mathrm{O}$ variations (Table 5).

Core 41-366-20, $\sim 177$ mbsf), which suggests that the specimens we observed at the base of Core 41-366-18 are reworked; and (6) an indication that the FO of Globorotalia peripheroacuta at Site 366 is delayed by a hiatus and that this level is about $13.9 \mathrm{Ma}$ at Site 366 vs. $14.9 \mathrm{Ma}$ elsewhere.

The above implications from a correlation of Site 366 to the Site 563 standard (Table 5) are based upon the assumption that the age estimates used for the standard section (Site 563) are correct. Any miscorrelation of the standard section to the GPTS would change the age estimates of Table 5. Despite these potential uncertainties, we think that integrating isotope stratigraphy with biostratigraphy in this manner provides an excellent means of independently testing biostratigraphic correlations.

A comparison of eastern equatorial Atlantic Sites 366 and 667 with western North Atlantic Site 563 isotope records
(Figs. 1, 7, and 8) also shows excellent agreement among records. General trends in the $\delta^{18} \mathrm{O}$ and $\delta^{13} \mathrm{C}$ records are similar, and the Oligocene/Miocene (24-23 Ma) and middle Miocene (14.8-13 Ma) $\delta^{18} \mathrm{O}$ increases are the same. The carbon isotope composition of the eastern and western basins were similar in certain intervals (late Oligocene-earliest Miocene, early middle Miocene, late Miocene); however, significant differences occurred during the earliest Oligocene, middle Oligocene, early Miocene, and late middle Miocene. The following summaries present the evolution of deep-water circulation in the Atlantic as inferred from this and previous studies.

\section{Earliest Oligocene}

The $\delta^{13} \mathrm{C}$ record for the interval from 36 to $34 \mathrm{Ma}$ at western North Atlantic Site $563\left(\right.$ mean $\delta^{13} \mathrm{C}$ values $=1.2 \%$; number of 
samples $[n]=12$ ) exhibited higher values than at Pacific Sites $77($ mean $=0.8 \% \circ ; n=19)$ and $574($ mean $=0.8 \% ; n=4$; Keigwin and Keller, 1984; Miller and Thomas, 1985). This indicates that nutrient-depleted NC/NADW was supplied to the western North Atlantic, which is consistent with seismic stratigraphic evidence for a northern source of bottom water at this time (Miller and Tucholke, 1983).

Carbon isotope values for Site 366 for the interval from 36 to $34 \mathrm{Ma}$ (mean $=0.9 \% ; n=6$ ) are intermediate between the western Atlantic and Pacific locations. This suggests that the western Atlantic may have been better ventilated than the eastern North Atlantic at this time; however, eastern basin Site 522 (4441 m present depth; $\sim 3000 \mathrm{~m}$ paleodepth) was similar in $\delta^{13} \mathrm{C}$ composition to Site 563 during the earliest Oligocene (mean value for $36-34 \mathrm{Ma}=1.2 \% ; n=24$; Miller et al., 1988), while eastern basin Site 529 (3035 m present depth; 2400-2600 m paleodepth) was slightly depleted in ${ }^{13} \mathrm{C}$ relative to Site 563 during this interval (mean value $=1.0 \%$; $n$ =9; Miller, unpubl. data). Boersma et al. (1987) also noted that the South Atlantic (both eastern and western basins) was filled with waters with high $\delta^{13} \mathrm{C}$ values during the earliest Oligocene.

The data from all locations has one important tectonic implication: ${ }^{13} \mathrm{C}$-enriched (relative to the Pacific) bottom waters were able to enter the eastern basin and therefore could not have been a tectonic blockage. We suggest the following scenario for the earliest Oligocene:

1. Young ( ${ }^{13} \mathrm{C}$-enriched, nutrient-depleted, high oxygen) bottom waters similar to NC/NADW entered the North Atlantic via overflow from the Greenland-Scotland Ridge (e.g., Miller and Tucholke, 1983; Miller et al., 1987a; Miller and Wright, in press).

2. These waters filled the western basins of the South Atlantic and penetrated through the equatorial fracture zones into the deep eastern basins ( $>3000 \mathrm{~m}$; e.g., Site 522).

3. Shallower locations in the eastern basin (Sites 366 and 529) may have been influenced by mixing with an intermediate water mass with lower $\delta^{13} \mathrm{C}$ values.

\section{"Middle", and Late Oligocene}

Very low $\delta^{13} \mathrm{C}$ values $(\sim 0.0 \%$ o $)$ occurred in the eastern basin locations from $\sim 33$ to $30 \mathrm{Ma}$ (Fig. 7). (At Site $366, \delta^{13} \mathrm{C}$ values were high from 34 to $33 \mathrm{Ma}$, but the coeval Site 667 had low $\delta^{13} \mathrm{C}$ values; this uncertainty may be due to biostratigraphic uncertainties; we have not yet tuned the records.) The interval from 33 to $30 \mathrm{Ma}$ is not well represented in the western basins due to a hiatus at Site 563 (Fig. 7). Still, the "middle" Oligocene $\delta^{13} \mathrm{C}$ values noted at eastern basin locations (Sites 366, 667, and 119; see Miller and Curry, 1982) were the lowest in the world at this time and undoubtedly reflect low ventilation of the eastern basins.

In the interval from 30 to $25 \mathrm{Ma}, \delta^{13} \mathrm{C}$ composition in the eastern Atlantic (Sites 366 and 667; see also Site 522) was similar to that in the western basin (Fig. 7; Miller et al., 1988) and similar to Pacific locations (Sites 77 and 574; see Miller and Fairbanks, 1985). The uniform $\delta^{13} \mathrm{C}$ composition of the Atlantic and deep Pacific suggests that NC/NADW deepwater circulation was reduced, probably for the entire interval from 33 to $25 \mathrm{Ma}$. This interpretation is supported by seismic stratigraphic evidence for reduced circulation at this time (Miller and Tucholke, 1983).

\section{Latest Oligocene-Earliest Miocene}

Carbon isotope values during this interval (25-23 Ma) were similar at eastern and western basin locations (Fig. 7; Sites 366,563 , and 667); all Atlantic locations were enriched in ${ }^{13} \mathrm{C}$ relative to the Pacific (see Miller and Fairbanks, 1985). This suggests that the western North Atlantic and the eastern basins were flooded with ${ }^{13} \mathrm{C}$-enriched NC/NADW, which originated in the high northern latitudes through overflow across the Greenland-Scotland Ridge.

\section{Early Miocene}

The western North Atlantic was still enriched in ${ }^{13} \mathrm{C}$ relative to the Pacific in the early Miocene, reflecting a continued supply of nutrient-depleted water (NC/NADW) to the North Atlantic (Miller and Fairbanks, 1985). Carbon isotope values at western basin Site 563 were slightly higher than at the eastern basin sites (Figs. 7 and 8). Miller et al. (1987a) similarly note that eastern basin Site 608 was lower in $\delta^{13} \mathrm{C}$ than Site 563 in the early Miocene; however, the magnitude of the difference between Sites 366/667 and Site 563 is less than reported by Miller et al. (1987a). We ascribe eastern to western basin $\delta^{13} \mathrm{C}$ differences to slightly lower ventilation of the eastern basins during the early Miocene.

\section{Early Middle Miocene}

The Atlantic (Site 563) to Pacific (Sites 77 and 289) $\delta^{13} \mathrm{C}$ differences during the early middle Miocene (16-14 Ma) were negligible, suggesting that the NC/NADW supply had greatly diminished (Miller and Fairbanks, 1985). Carbon-isotope values from Sites 366, 563, and 667 were also similar at this time, with less than a $0.25 \%$ difference (Figs. 7 and 8). The convergence of $\delta^{13} \mathrm{C}$ isotope values probably reflected one source of deep-water production at this time: the Southern Ocean.

\section{Late Middle Miocene}

There was a large Atlantic (Site 563) to Pacific (Sites 77 and 289) $\delta^{13} \mathrm{C}$ difference $(>0.5 \%$ ) during the late middle Miocene (about 14-10 Ma; Miller and Fairbanks, 1985). This has been interpreted as reflecting a high supply of NC/NADW to the western North Atlantic (Miller and Fairbanks, 1985), although Southern Ocean records are needed to confirm this. Miocene $\delta^{13} \mathrm{C}$ values at Site 366 were lower than at Sites 563 and 667 between about 12.5 and $10 \mathrm{Ma}$ (Fig. 7). The significance of this is unclear, for $\delta^{13} \mathrm{C}$ values at Site 667 are similar to those at Site 563 (Fig. 7). It is possible that this reflects a real difference in water masses at Site 366 vs. Site 667 , but more data are required to verify this interval of low $\delta^{13} \mathrm{C}$ values at Site 366 .

\section{Early Late Miocene}

Moderate offsets between Atlantic and Pacific $\delta^{13} \mathrm{C}$ values occurred from about 10 to $8.5 \mathrm{Ma}$ (Miller and Fairbanks, 1985). There are insufficient data to reconstruct late Miocene eastern to western basin $\delta^{13} \mathrm{C}$ differences (Fig. 7).

\section{CONCLUSIONS}

The use of Cenozoic stable isotope stratigraphy has not been fully appreciated; we demonstrate that stable isotopes provide stratigraphic control independent of biostratigraphic problems. The well-known earliest Oligocene and middle Miocene $\delta^{18} \mathrm{O}$ increases have been well established as chronostratigraphic markers; we also note that a sharp $\delta^{18} \mathrm{O}$ increase occurred near the end of the Oligocene, providing another marker. In addition to these large $(>1.0 \%) \delta^{18} \mathrm{O}$ variations, smaller $(\sim 0.5 \%) \delta^{18} \mathrm{O}$ fluctuations can be used to improve correlations. For example, we have found that there are at least eight recognizable Miocene $\delta^{18} \mathrm{O}$ fluctuations (Table 5) that can be used for potential correlations. These fluctuations have not been fully tied to the GPTS nor have they been documented in all oceans. Ties into the GPTS can be improved through analyses of the best available magneto- 
stratigraphic sections (e.g., Site 608; Clement and Robinson, 1987; Miller et al., 1987a).

Carbon isotope comparisons continue to be one of the strongest tools for reconstructing deep/bottom-water changes, yet the history of such changes continues to be controversial. For example, Woodruff and Savin (1989) used $\delta^{13} \mathrm{C}$ and benthic foraminifer faunal data to develop a scenario of deep-water history that is different from ours: (1) little influence of NC/NADW during the early Miocene, (2) moderate influence during the middle Miocene, and (3) virtually modern influence during the late Miocene. Variations in NC/NADW supply to the Atlantic apparently occurred during the early Oligocene through Miocene, and depending upon the data used, different investigators have come to different conclusions regarding the presence or absence of NC/NADW during the pre-middle Miocene (cf. Schnitker, 1980, and Miller and Tucholke, 1983).

We suggest that the carbon (Miller and Fairbanks, 1985, and this study) and seismic stratigraphic (Miller and Tucholke, 1983) evidence indicates the presence of pre-middle Miocene $\mathrm{NC} / \mathrm{NADW}$, but that the supply of NC/NADW was generally lower during the pre-middle Miocene than at present or during the Pliocene (e.g., Miller and Curry, this vol.). Reconstructions of NC/NADW flux can be made using the Southern Ocean as a monitor of NC/NADW flux (Oppo and Fairbanks, 1987); however, the eastern basins also provide a measure of the relative contributions of NC/NADW and AABW at the sills to the eastern basin (Oppo and Fairbanks, 1987). Using this Quaternary model and the data presented here, we suggest that the relative flux of NC/NADW to the eastern basin was (1) moderate during the early Oligocene; (2) low to nil during the "middle" Oligocene; (3) moderate during the latest Oligocene to earliest Miocene; (4) low during the early Miocene and early middle Miocene; and (5) moderate to high during the late middle Miocene.

Our data are not sufficient to constrain the late Miocene history of the eastern basins. The absolute flux of NC/NADW to the Atlantic may be improved by using carbon isotope records from the Southern Oceans (Oppo and Fairbanks, 1987). Future studies of Miocene deep-water history will focus on $\delta^{13} \mathrm{C}$ from the Southern Oceans (e.g., Wright et al., 1988).

\section{ACKNOWLEDGMENTS}

We thank W. A. Berggren, M. E. Katz, D. V. Kent, E. Thomas, and an anonymous reviewer for reviews; the shipboard party of Leg 108 for allowing us to sample Hole 667 ; M. E. Katz for technical assistance; R. K. Olsson for discussions of the taxonomy of Globorotalia kugleri; and N. Katz for drafting the figures. Samples were provided by the Ocean Drilling Program. Research was supported by National Science Foundation Grants OCE85-21690 and OCE8700005 . This is Lamont-Doherty Geological Observatory Contribution No. 4470 .

\section{REFERENCES}

Berger, W. H., and Winterer, E. L., 1974. Plate stratigraphy and the fluctuation carbonate line. In Hsü, K. J., and Jenkins, H. C. (Eds.), Pelagic Sediments on Land and under the Sea. Internat. Assoc. Sedimentol., Spec. Publ., 1:11-48.

Berggren, W. A., Kent, D. V., and Flynn, J. J., 1985a. Jurassic to Paleogene: Part 2, Paleogene geochronology and chronostratigraphy. In Snelling, N. J. (Ed.), The Chronology of the Geological Record. Geol. Soc. Mem. (London), 10:141-195.

Berggren, W. A., Kent, D. V., and Van Couvering, J. A., 1985b. Neogene: Part 2, Neogene geochronology and chronostratigraphy. In Snelling, N. J. (Ed.), The Chronology of the Geological Record. Geol. Soc. Mem. (London), 10:211-260.

Berggren, W. A., and Miller, K. G., 1988. Paleogene tropical planktonic foraminiferal biostratigraphy and magnetobiochronology. Micropaleontology 34:362-380.
Blow, W. H., 1969. Late middle Eocene to Recent planktonic foraminiferal biostratigraphy. In Brönnimann, P., and Renz, H. H. (Eds.), First International Conference on Planktonic Microfossils, 1967, Geneva: Leiden (E. J. Brill), 1:199-421.

Blow, W. H., 1979. The Cainozoic Globigerinida: London (E. J. Brill).

Boersma, A., and Shackleton, N. J., 1978. Oxygen and carbon isotope record through the Oligocene, DSDP Site 366, equatorial Atlantic. In Lancelot, Y., Seibold, E., et al., Init. Repts. DSDP, 41: Washington (U.S. Govt. Printing Office), 957-962.

Boersma, A., Premoli Silva, I., and Shackleton, N. J., 1987. Atlantic Eocene planktonic foraminiferal paleohydrographic indicators and stable isotope paleoceanography. Paleoceanography, 2:287-331.

Bolli, H. M., 1957. Planktonic foraminifera from the OligoceneMiocene Cipero and Lengua Formations of Trinidad, B.W.1. U.S Nat. Mus. Bull., 215:97-123.

Bolli, H. M., and Saunders, J. B., 1985. Oligocene to Holocene low latitude planktic foraminifera. In Bolli, H. M., Saunders, J. B., and Perch-Nielsen, K. (Eds.), Plankton Stratigraphy: Cambridge (Cambridge Univ. Press), 155-262.

Bonatti, E., and Chermak, A., 1981. Formerly emerging crustal blocks in the equatorial Atlantic. Tectonophysics, 72:165-180.

Broecker, W. S., and Peng, T.-H., 1982. Tracers in the Sea: Palisades, NY (Eldigio Press).

Bukry, D., 1978. Cenozoic coccolith and silicoflagellate stratigraphy, offshore northwest Africa, Deep Sea Drilling Project, Leg 41. In Lancelot, Y., Seibold, E., et al. (Eds.), Init. Repts. DSDP, 41: Washington (U.S. Govt. Printing Office), 689-708.

Clement, B., and Robinson, F., 1987. The magnetostratigraphy of Leg 94 sediments. In Ruddiman, W. F., Kidd, R. B., Thomas, E., (Eds.), Init. Repts. DSDP, 94, Pt. 2: Washington (U.S. Govt. Printing Office), 635-650.

Curry, W. B., and Crowley, T. J., 1987. The $\delta^{13} \mathrm{C}$ of equatorial Atlantic surface waters: implications for Ice Age $\mathrm{pCO}_{2}$ levels. Paleoceanography, 2:489-517.

Curry, W. B., and Lohmann, G. P., 1983. Reduced advection into Atlantic Ocean deep eastern basins during last glacial maximum. Nature, 306:577-580.

1985. Carbon deposition rates and deep water residence time in the equatorial Atlantic Ocean throughout the last 160,000 years. In Sundquist, E. T., and Broecker, W. S. (Eds.), The Carbon Cycle and Atmospheric $\mathrm{CO}_{2}$ : Natural Variations Archean to Present: Washington (American Geophysical Union), 32:285-301.

1986. Late Quaternary carbonate sedimentation at the Sierra Leone Rise (eastern equatorial Atlantic Ocean). Mar. Geol., 70:223-250.

Fairbanks, R. G., and Matthews, R. K., 1978. The marine oxygen isotopic record in Pleistocene coral, Barbados, West Indies. Quat. Res., 10:181-196.

Graham, D. W., Corliss, B. H., Bender, M. L., and Keigwin, L. D., 1981. Carbon and oxygen isotopic disequilibria of Recent benthic foraminifera. Mar. Micropaleontol., 6:483-497.

Haq, B. U., Worsley, T. R., Burckle, L. H., Douglas, R. G., Keigwin, L. D., Jr., et al., 1980. Late Miocene marine carbon-isotopic shift and synchroneity of some phytoplanktonic biostratigraphic events. Geology, 8:427-431

Hodell, D. A., and Kennett, J. P., 1986. Late Miocene-early Pliocene stratigraphy and paleoceanography of the South Atlantic and Southwest Pacific Oceans: a synthesis. Paleoceanography, 1:285312 .

Imbrie, J., Hays, J., Martinson, D. G., McIntyre, A., Mix, A. C., Morley, J. J., Pisias, N. G., Prell, W. L., and Shackleton, N. J., 1984. The orbital theory of Pleistocene climate: support from a revised chronology of the marine $\delta^{18} \mathrm{O}$ record. In Berger, A. L., Imbrie, J., Hays, J., Kukla, G., and Saltzman, B. (Eds.), Milankovitch and Climate (Pt. 1): Dordrecht (D. Reidel), 269-305.

Johnson, D. A., and Nigrini, C. A., 1985. Time-transgressive late Cenozoic radiolarian events of the equatorial Indo-Pacific. Science, 230:538-540.

Keigwin, L. D., 1980. Paleoceanographic change in the Pacific at the Eocene-Oligocene boundary. Nature, 287:722-725.

1987. Toward a high-resolution chronology for the latest Miocene paleoceanographic events. Paleoceanography, 2:639680 . 
Keigwin, L. D., and Keller, G., 1984. Middle Oligocene climate change from equatorial Pacific DSDP Site 77. Geology, 12:16-19.

Keigwin, L. D., Aubrey, M.-P., and Kent, D. V., 1987. North Atlantic late Miocene stable-isotope stratigraphy, biostratigraphy, and magnetostratigraphy. In Ruddiman, W. F., Kidd, R. B., Thomas, E. (Eds.), Init. Repts. DSDP, 94, Pt. 2: Washington (U.S. Govt. Printing Office), 935-963.

Keller, G., 1986. Stepwise mass extinctions and impact events: late Eocene to early Oligocene. Mar. Micropaleontol., 10:267-293.

Kennett, J. P., and Shackleton, N. J., 1976. Oxygen isotope evidence for the development of the psychrosphere 38 m.y. ago. Nature, 260:513-515.

Kennett, J. P., and Srinivasan, M. S., 1985. Neogene Planktonic Foraminifera: Stroudsburg, PA (Hutchinson Ross).

Kidd, R. B., and Ramsey, A.T.S., 1987. The geology and formation of the King's Trough Complex in the light of Deep Sea Drilling Project Site 608 Drilling. In Ruddiman, W. F., Kidd, R. B., Thomas, E. (Eds.), Init. Repts. DSDP, 94, Pt. 2: Washington (U.S. Govt. Printing Office), 1245-1261.

Krasheninnikov, V. A., and Pflaumann, U., 1978a. Zonal stratigraphy and planktonic foraminifers of Paleogene deposits of the Atlantic Ocean to the west off Africa (Deep Sea Drilling Project, Leg 41). In Lancelot, Y., Seibold, E., et al. (Eds.), Init. Repts. DSDP, 41: Washington (U.S. Govt. Printing Office), 581-612.

1978b. Zonal stratigraphy of Neogene deposits of the eastern part of the Atlantic Ocean by means of planktonic foraminifers, Leg 41 Deep Sea Drilling Project. In Lancelot, Y., Seibold, E., et al. (Eds.), Init. Repts. DSDP, 41: Washington (U.S. Govt. Printing Office), 613-658.

Lamb, J. L., and Stainforth, R. M., 1976. Unreliability of Globigerinoides datum. AAPG Bull., 60:1564-1569.

Lancelot, Y., Seibold, E., et al., 1978. Init. Repts. DSDP, 41: Washington (U.S. Govt. Printing Office).

Metcalf, W. G., Heezen, B. C., and Stalcup, M. C., 1964. The sill depth of the Mid-Atlantic Ridge in the equatorial region. Deep-Sea Res., 11:1-10.

Miller, K. G., and Curry, W. B., 1982. Eocene to Oligocene benthic foraminiferal isotopic record in the Bay of Biscay, Nature, 296: $347-350$.

Miller, K. G., and Fairbanks, R. G., 1985. Oligocene-Miocene global carbon and abyssal circulation changes. In Sundquist, E., and Broecker, W. S. (Eds.), The Carbon Cycle and Atmospheric $\mathrm{CO}_{2}$ : Natural Variations Archean to Present: Washington (American Geophysical Union), 469-486.

Miller, K. G., and Thomas, E., 1985. Late Eocene to Oligocene benthic foraminiferal isotopic record, Site 574, equatorial Pacific. In Mayer, L., Theyer, F., et al. (Eds.), Init. Repts. DSDP, 85: Washington (U.S. Govt. Printing Office), 771-777.

Miller, K. G., and Tucholke, B. E., 1983. Development of Cenozoic abyssal circulation south of the Greenland-Scotland Ridge. In Bott, M.H.P., Saxov, S., Talwani, M., and Thiede, J. (Eds.), Structure and Development of the Greenland-Scotland Ridge: New York (Plenum Press), 549-589.

Miller, K. G., and Wright, J. D., in press. Subsidence history of the Greenland-Scotland Ridge revisited: implications of uplift on deep-water circulation history. 28th International Geological Congress, Washington.

Miller, K. G., Aubrey, M.-P., Kahn, M. J., Meillo, A. J., Kent, D. V., and Berggren, W. A., 1985a. Oligocene-Miocene biostratigraphy, magnetostratigraphy, and isotopic stratigraphy of the western North Atlantic. Geology, 13:257-261.

Miller, K. G., Curry, W. B., and Ostermann, D. R., 1985b. Late Paleogene (Eocene to Oligocene) benthic foraminiferal oceanography of the Goban Spur region, Deep Sea Drilling Project Leg 80. In de Graciansky, P. C., Poag, C. W., et al. (Eds.), Init. Repts. DSDP, 80: Washington (U.S. Govt. Printing Office), 505-538.

Miller, K. G., Fairbanks, R. G., and Thomas, E., 1987a. Benthic foraminiferal carbon isotopic records and the development of abyssal circulation in the eastern North Atlantic. In Ruddiman, W. F., Kidd, R. B., Thomas, E., et al. (Eds.), Init. Repts. DSDP, 94, Pt. 2: Washington (U.S. Govt. Printing Office), 981-996.

Miller, K. G., Fairbanks, R. G., and Mountain, G. S., 1987b. Tertiary oxygen isotope synthesis, sea-level history, and continental margin erosion. Paleoceanography, 2:1-19.
Miller, K. G., Janecek, T. R., Katz, M. E., and Keil, D., 1987c. Abyssal circulation and benthic foraminiferal changes near the Paleocene/Eocene boundary. Paleoceanography, 2:741-761.

Miller, K. G., Feigenson, M. D., Kent, D. V., and Olsson, R. K., 1988. Upper Eocene to Oligocene stable isotope ${ }^{87} \mathrm{Sr} /{ }^{86} \mathrm{Sr}, \delta^{18} \mathrm{O}$, $\left.\delta^{13} \mathrm{C}\right)$ standard section, Deep Sea Drilling Project Site 522. Paleoceanography, 3:223-233.

Mix, A. C., and Fairbanks, R. G., 1985. North Atlantic surface-ocean control of Pleistocene deep-ocean circulation. Earth Planet. Sci. Lett., 73:231-243.

Oberhänsli, H., and Toumarkine, M., 1985. The Paleogene oxygen and carbon isotope history of Sites 522, 523, and 524 from the central South Atlantic. In Hsü, K. J., and Weissert, H. J. (Eds.), South Atlantic Paleoceanography: Oxford (Oxford Univ. Press), 124-147.

Oppo, D., and Fairbanks, R. G., 1987. Variability in deep and intermediate water circulation during the past 25,000 years: Northern Hemisphere modulation of the southern ocean. Earth Planet. Sci. Lett., 86:1-15.

Pisias, N. G., Shackleton, N. J., and Hall, M. A., 1985. Stable isotope and calcium carbonate records from hydraulic piston cored Hole 574A: high-resolution records from the middle Miocene. In Mayer, L., Theyer, F., et al. (Eds.), Init. Repts. DSDP, 85: Washington (U.S. Govt. Printing Office), 735-748.

Pomerol, C., and Premoli Silva, I., 1986. The Eocene-Oligocene transition: events and boundary. In Pomerol, C., and Premoli Silva, I. (Eds.), Terminal Eocene Events: Amsterdam (Elsevier), 1-24.

Poore, R. Z., and Matthews, R. K., 1984. Late Eocene-Oligocene oxygenand carbon-isotope record from South Atlantic Ocean, Deep Sea Drilling Project Site 522. In Hsü, K. J., et al. (Eds.), Init. Repts. DSDP, 73: Washington (U.S. Govt. Printing Office), 725-735.

Ruddiman, W. F., Raymo, M., and McIntyre, A., 1986. Matuyama 41,000-year cycles: North Atlantic Ocean and Northern Hemisphere ice sheets. Earth Planet. Sci. Lett., 80:117-129.

Ruddiman, W., Sarnthein, M., et al., 1988. Proc. ODP, Init. Repts., 108: College Station, TX (Ocean Drilling Program).

Savin, S. M., Douglas, R. G., and Stehli, F. G., 1975. Tertiary marine paleotemperatures. Geol. Soc. Am. Bull., 86:1499-1510.

Schnitker, D., 1980. Global paleoceanography and its deep-water linkage to the Antarctic glaciation. Earth-Sci. Rev., 16:1-20.

Sclater, J. G., Anderson, R. N., and Bell, N. L., 1971. Elevation of ridges and evolution of the central eastern Pacific. J. Geophys. Res., 76:7888-7915.

Shackleton, N. J., 1987. The carbon isotope record of the Cenozoic: history of organic carbon burial and of oxygen in the ocean and atmosphere. In Brooks, J., and Fleet, A. J. (Eds.), Marine Petroleum Source Rocks (Vol. 26): Oxford (Blackwell Scientific), 423-434.

Shackleton, N. J., Hall, M. A., and Boersma, A., 1984. Oxygen and carbon isotope data from Leg 74 foraminifers. In Mayer, L., Theyer, F., et al. (Eds.), Init. Repts. DSDP, 74: Washington (U.S. Govt. Printing Office), 599-612.

Shackleton, N. J., Imbrie, J., and Hall, M. A., 1983. Oxygen and carbon isotope record of East Pacific core V19-30: implications for the formation of deep water in the late Pleistocene. Earth Planet. Sci. Lett., 65:233-244.

Shackleton, N. J., and Kennett, J. P., 1975. Paleotemperature history of the Cenozoic and the initiation of Antarctic glaciation: oxygen and carbon isotopic analyses in DSDP Sites 277, 279, and 281. In Kennett, J. P., Houltz, R. E., et al. (Eds.), Init. Repts. DSDP, 29; Washington (U.S. Govt. Printing Office), 743-755.

Shackleton, N. J., and Opdyke, N. D., 1973. Oxygen isotopic and palaeomagnetic stratigraphy of equatorial Pacific core V28-238: oxygen isotope temperatures and ice volume on a $10^{5}$ and $10^{6}$ year scale. Quat. Res., 3:39-55.

Snyder, S. W., Muller, C., and Miller, K. G., 1985. Biostratigraphy and paleoceanography across the Eocene/Oligocene boundary at Deep Sea Drilling Project Site 549. In de Graciansky, P. C., Poag, C. W., et al. (Eds.), Init. Repts. DSDP, 80: Washington (U.S. Govt. Printing Office), 567-572.

Stainforth, R. M., Lamb, J. L., Luterbacher, H., Beard, J. H., and Jeffords, R. M., 1975. Cenozoic Planktonic Foraminiferal Zonation and Characteristics of Index Fossils. Univ. Kans. Paleontol. Contrib., Article 62.

Vergnaud-Grazzini, C. and Oberhänsli, H., 1986. Isotopic events at the Eocene-Oligocene transition: a review. In Pomerol, C., and 
Premoli Silva, I. (Eds.), Terminal Eocene Events: Amsterdam (Elsevier), 311-330.

Vincent, E., and Berger, W. H., 1985. Carbon dioxide and polar cooling in the Miocene: the Monterey Hypothesis. In Sundquist, E. T., and Broecker, W. S. (Eds.), The Carbon Cycle and Atmospheric $\mathrm{CO}_{2}$ : Natural Variations Archean to Present: Washington (American Geophysical Union), 32:455-468.

Woodruff, F., Savin, S. M., and Douglas, R. G., 1981. A Miocene stable isotopic record: a detailed deep Pacific Ocean study and its paleoclimatic implications. Science, 212:665-668.

Woodruff, F. and Savin, S. M., 1989. Miocene deep-water oceanography. Paleoceanography, 4:87-140.

Wright, J. D., Miller, K. G., and Fairbanks, R. G., 1988. Evolution of Miocene deep-water circulation: carbon-isotope contributions to the eastern Atlantic and Southern Oceans. Eos, 69:381.

Date of initial receipt: 21 July 1988 Date of acceptance: 21 October 1988 Ms 108B-177

\section{TAXONOMIC AND STRATIGRAPHIC NOTES}

\section{Globorotalia kugleri Bolli}

In this study, we employed a sensu stricto definition of $G$. kugleri Bolli, 1957 ([5], p. 118, pl. 28, figs. 5a-6). We follow Blow's (1969) tripartite subdivision of the $G$. kugleri plexus into three distinct species: $G$. mendacis, $G$. pseudokugleri, and $G$. kugleri. We restrict the concept of $G$. kugleri s.s. to forms with distinctly recurved spiral intercameral sutures and an open well-defined deep umbilical pit and a distinct, well-developed lip that borders the pit, and to forms that are generally rather flat on the spiral side approaching the point where the chambers take on a plano-convex appearance in axial view.

In contrast, $G$. mendacis has less recurved spiral sutures; generally less embracing chambers in the spiral view; a shorter, more closed umbilicus (sometimes with a small, reduced final chamber); and a less distinct lip bordering the umbilicus. It is inflated on the spiral side so that its test takes on a more equally biconvex form in the axial view.

In comparing $G$. mendacis with $G$. pseudokugleri, the former has more curved spiral intercameral sutures. Globorotalia mendacis has weakly recurved sutures, whereas G. pseudokugleri has only curved, but not recurved sutures. We disagree with Blow (1969) on this point, for Blow describes $G$, pseudokugleri as having recurved spiral intercameral sutures, a more closed umbilicus, more embracing yet less inflated chambers, with a subacute biconvex appearance in the axial view.

In suggesting that the morphologic characters of $G$. mendacis are intermediate between those of $G$. pseudokugleri and G. kugleri, Blow (1969) implied that it is easier to differentiate between these latter two forms. In contrast, we find that separating $G$. pseudokugleri from $G$. kugleri has proven to be the most difficult and has led to considerable confusion in recognizing the Oligocene/Miocene boundary in published studies. In comparing these two forms, we find that the major feature distinguishing them is the nature of the intercameral sutures: those of $G$. kugleri are strongly recurved, while those of $G$. pseudokugleri are merely curved (not recurved as Blow, 1969, suggested). Other, less diagnostic differences are: (1) G. pseudokugleri has a rounded peripheral margin whereas that of $G$. kugleri is markedly plano-convex in the axial view due to its nearly flat spiral side; (2) the umbilicus of $G$. pseudokugleri is open and deep as in G. kugleri but features a slightly less distinct pit; (3) there is a distinct lip bordering the umbilicus of both forms; and (4) G. pseudokugleri, in contrast to G. kugleri, has inflated, subglobular chambers.

The clear, consistent differentiation between $G$. kugleri and $G$. pseudokugleri is critical, for the identification of $G$. kugleri in a values vs. sensu lato sense has significant implications for recognition of the Oligocene/Miocene boundary. We follow Berggren et al. (1985a, $1985 \mathrm{~b}$ ) in recognizing the Oligocene/Miocene boundary by the FO of $G$. kugleri. Since G. pseudokugleri appeared several million years before G. kugleri ( $30 \mathrm{~m}$ below the FO of $G$. kugleri at Hole 366 ; approximately $2.7 \mathrm{~m}$. y.; Fig. 4), misidentification can result in serious miscorrelation among sites.

\section{Paragloborotalia opima opima (Bolli)}

We follow Bolli and Saunders (1985) in distinguishing between $P$. opima opima and $P$. opima nana on the basis of size: the former is restricted to four-chambered specimens $>0.39 \mathrm{~mm}(390 \mu \mathrm{m})$. We have found that the LO of $P$. opima opima is useful for recognizing the top of Zone P21b. However, we differ from Bolli (1957) and Bolli and Saunders (1985) in that we have found specimens of $P$. opima opima in strata immediately overlying strata with Pseudohastigerina at Sites 558 and 563 (Miller et al., 1985a), effectively allowing Bolli's G. opima opima Zone to encompass his Globigerina ampliapertura Zone. At Site 366 , four-chambered forms have a FO at the base of Hole $366 \mathrm{~A}$, while five-chambered forms $(\sim 375 \mu \mathrm{m})$ transitional to $P$. opima opima (Bolli and Saunders, 1985) appeared immediately above the LO of Pseudohastigerina spp. (Fig. 4).

\section{Paragloborotalia opima nana (Bolli)}

We note the LO of this species at the same level as the LO of Globorotalia kugleri ( = top Zone N4) at Site 366 . We note the LO of this species at North Atlantic Site 563 between Sections 82-563A$15 \mathrm{H}-2,117-123 \mathrm{~cm}$, and $85-563 \mathrm{~A}-14 \mathrm{H}-5,114-120 \mathrm{~cm}$, in Magnetochron C6Ar and within the range of G. kugleri. Other authors (e.g., Bolli and Saunders, 1985) have suggested that this taxon became extinct within the late Oligocene; we show here that it persisted into the earliest Miocene. We think that the LO of $P$. opima nana will prove to be a useful marker of the earliest Miocene.

\section{Globigerinatella insueta Cushman and Stainforth}

Because of its sporadic distribution, and notwithstanding its distinctive morphology, this species constitutes an undesirable zonal marker. Its FO is used as the marker for Bolli's (1957) Catapsydrax stainforthi Zone and Blow's (1979) Zone N6. We have found that it is rare in the lower part of its range; for example, at Site 563, it does not appear until Zone N8 (Miller et al., 1985a).

One other taxon that may be suitable for subdividing this middle lower Miocene interval (i.e., between the LO of G. kugleri and the LO of Catapsydrax dissimilis; Zones N5-N6) is the FO of Globorotalia praescitula. Bolli and Saunders (1985) indicate that this taxon first occurred in the earliest $C$. dissimilis Zone $(=\mathrm{N} 5)$, immediately after the LO of G. kugleri. Berggren et al. (1985b) suggest that $G$. praescitula appeared in late Chron C5Dn, at about the same time as the LO of $C$. dissimilis (= base of Zone N7). However, at Sites 667 (Fig. 5), 558, and 563 (Miller et al., 1985a), we have found that $G$. praescitula appeared essentially midway between the LO of $G$. kugleri and the LO of $C$. dissimilis.

The calibrations of the FO vs, the magnetostratigraphy at Sites 558 and 563 are tenuous because of a hiatus inferred immediately below the FO at Hole 558 and immediately above the FO at Hole 563; at Site 558 the FO is within Zone NN2, whereas at Site 563 it is in Zone NN4 (Miller et al., 1985a). Despite these uncertainties, we propose that further study may show that this taxon is useful in subdividing undifferentiated Zones N5-N6. To support this, Kennett and Srinivasan (1985) note the FO of G. praescitula in the late part of Zone N5. 